CU-TP-598, LBL-32682

\title{
Multiple Collisions and Induced Gluon Bremsstrahlung in QCD 尚
}

\author{
Miklos Gyulassy \\ Physics Department, Columbia University, New York, NY 10027 \\ and \\ Xin-nian Wang \\ Nuclear Science Division, MS 70A-3307, Lawrence Berkeley Laboratory \\ University of California, Berkeley, CA 94720
}

September 25, 2018

\begin{abstract}
Induced soft gluon bremsstrahlung associated with multiple collisions is calculated via perturbative QCD. We derive the non-abelian analog of the LandauPomeranchuk effect that suppresses induced soft radiation with formation times exceeding the mean free path, $\lambda$. The dependence of the suppression effect on the $S U(N)$ representation of the jet parton as well as the kinematic variables is expressed through a radiation formation factor. Unlike in QED, the finite contribution from the small $x$ regime in QCD leads to an approximately constant radiative energy loss per unit length, $d E / d z \propto \mu^{2}$, in the high energy limit that is sensitive to the infrared screening scale, $\mu$, of the medium. As a function of the dimensionless parameter $\zeta=\lambda \mu^{2} / E$, we show furthermore how the energy dependence of $d E / d z$ evolves from the above constant for $\zeta \ll 1$ to the more familiar (Bethe-Heitler) linear dependence for $\zeta \gg 1$.
\end{abstract}

\footnotetext{
* This work was supported by the Director, Office of Energy Research, Division of Nuclear Physics of the Office of High Energy and Nuclear Physics of the U.S. Department of Energy under Contract Nos. DE-AC03-76SF00098, DE-FG05-90ER40592, and DE-FG02-93ER40764.
} 


\section{Introduction}

Radiative energy loss of ultra-relativistic particles passing through dense matter is of interest not only because of its many practical applications, but also because it illustrates a characteristic destructive interference phenomenon caused by the finite formation time 1, 2],

$$
\tau(k) \sim \hbar / \Delta E(k) \sim 2 \omega / k_{\perp}^{2} \sim 2 / \omega \theta^{2}
$$

of quanta with large four momentum, $k^{\mu}=\left(\omega, k_{z}, \mathbf{k}_{\perp}\right)$, emitted at small angles, $\theta \approx k_{\perp} / \omega$, relative to the incident particle. In effect, $\tau(k)$, is the minimal time needed to resolve the transverse wavepacket of the quanta with, $\Delta x_{\perp} \sim \hbar / k_{\perp}$, from the wavepacket of its high energy $\left(E_{0} \gg \omega\right)$ parent. Destructive interference between radiation amplitudes associated with multiple collisions can be expected when the mean free path, $\lambda$, is short compared to the formation time. When $\tau(k) \gg \lambda$, the emitted quanta cannot resolve different elastic scattering centers, and the assumption of independent contributions from each separate scattering in the medium breaks down. This effect, first studied in QED and then in other field theories, is often referred to as the Landau-Pomeronchuck-Migdal (LPM) effect [1, 2].

Interest in analogous destructive interference phenomena in non-abelian theories is connected with attempts to understand the weak nuclear dependence of hard QCD processes and the use of those dependencies as a probe of the space-time development of hadronization. A high energy quark or gluon passing through dense (QCD) matter of course suffers multiple interactions. However, in the $Q \rightarrow \infty$ limit, QCD factorization theorems [3] apply that show that, as in the QED case, the soft radiation is emitted only from the external legs 4 . There are a large number of phenomena such as the very weak nuclear dependence of Drell-Yan yields 5, 6] and the apparent nuclear independence of very high energy quark fragmentation [7, 8], that confirms this basic factorization feature of asymptotic pQCD. As shown in Ref. [5], however, the assumptions leading to the factorization theorem break down for sub-asymptotic conditions. In the Drell-Yan process, for example, final state interaction corrections 
become important for sufficiently large nuclei with, $A^{2 / 3} \Lambda_{Q C D}^{2}>M_{D Y}^{2}$. In deep inelastic hadroporduction, nuclear dependence always exits in the $x_{F}<0$ region and disappears in the $x_{F}>0$ region only when $\kappa R / \nu \rightarrow 0$, where $\kappa \approx 1 \mathrm{GeV} / \mathrm{fm}[7,8]$.

In order to understand such nuclear effects more quantitatively, it is necessary to study induced radiation patterns associated with multiple collisions in QCD. Rough estimates for the magnitude of the suppression of non-abelian induced radiation have been made in [9, 11, 12, 13, 10]. The estimates for $d E / d z$ vary, however, widely ranging from energy independent [10], logarithmic energy dependent [9] to $d E / d z \propto E$ [12, 13. However, a detailed study of the LPM effect in QCD has not been performed to our knowledge in the context of multiple collision theory, taking into account essential non-abelian features of the problem. The aim of this paper is to initiate such a study.

While pQCD can serve only as a qualitative guide because the effective coupling, $\alpha_{s}$, is small only in extremely dense matter, e.g., a quark-gluon plasma at temperatures $T \gg T_{c} \sim \Lambda_{Q C D}$, it is instructive to explore its consequences in situations where approximations to multiple scattering can be used to simplify the problem. Additional motivation for this work is to compare radiation patterns due to multiple collision physics in pQCD with those suggested by phenomenological string models [8, 14, 15] for high energy $e A, p A$, and $A A$ reactions. We show, for example, that some features of the induced soft gluon rapidity distributions in pQCD are similar to the hadron distributions predicted by multi-string Lund type models utilizing string breaking and flip mechanisms $[8]$. In particular the effective string tension, $\kappa \sim 1 \mathrm{GeV} / \mathrm{fm}$ in those models is analogous to constant radiative energy loss due to induced soft radiation in the $x \ll 1$ regime in non-abelian multiple collision theory. Furthermore, additivity of radiation from multiple scatterings is limited to a domain $x<\kappa \lambda / E_{0}$, that shrinks as $E_{0}$ increases, just as in string flip models for multiple interactions [8]. However, in pQCD, unlike in string models, $\kappa=d E / d z \propto \mu^{2} \sim g^{2} T^{2}$, is found to be sensitive to the infrared screening scale, $\mu$, in the medium. Recall that other so called "string effects" were also found to arise naturally from interfering pQCD amplitudes for three jet events in $e^{+} e^{-}$[3]. Finally, we note the importance of clarifying radiative energy 
loss mechanisms and interference phenomena associated with formation zone physics for the further development of QCD transport theory and parton cascade models 16.

Before turning to the non-abelian problem, we recall briefly how the destructive interference for induced radiation occurs in QED. The Fourier transform of the current of a classical particle undergoing $m$ collisions at space-time points $x_{i}^{\mu}$ at which the four momenta change from $p_{i-1}^{\mu}$ to $p_{i}^{\mu}$ is

$$
J^{\mu}(k)=\sum_{i=1}^{m} J_{i}^{\mu}(k)=i e \sum_{i=1}^{m} e^{i k x_{i}}\left(\frac{p_{i}^{\mu}}{k p_{i}}-\frac{p_{i-1}^{\mu}}{k p_{i-1}}\right) .
$$

The resulting soft bremsstrahlung spectrum is given by

$$
\omega \frac{d^{3} n_{\gamma}}{d^{3} k}=\sum_{\epsilon}|\epsilon J(k)|^{2} /\left(2(2 \pi)^{3}\right)=-|J(k)|^{2} /\left(2(2 \pi)^{3}\right) \text {. }
$$

The sum contains $m$ diagonal terms, where the phase factors drop out, and $m(m-1)$ off diagonal terms involving phase factors, $\exp \left(i k\left(x_{i}-x_{j}\right)\right)$. Two extreme limits are obvious. One is the incoherent limit where $k\left(x_{i}-x_{j}\right) \gg 1$. In this case, the off diagonal phase factors tend to average to zero. This corresponds to the usual BetheHeitler limit, in which the radiated energy loss, $d E / d z=-E / L_{r}$, grows linearly with energy with $L_{r}$ being the radiation length (the ratio of the mean free path to the average fraction of the energy radiated per collision). The other extreme limit, is the one corresponding to $k\left(x_{i}-x_{j}\right) \ll 1$ for all $i, j$. In this case, all the phase factors are approximately unity, and there is an exact cancellation between adjacent terms in eq. (2). Only the radiation from initial and final lines contributes. This is the so-called the "Factorization limit" since the amplitude for soft radiation factors into an amplitude for multiple collisions times a current element depending only on the momenta of the external lines entering and leaving the reaction. Because the radiation intensity in the high $Q^{2}=-\left(p_{f}-p_{i}\right)^{2}$ limit increases only as $\log \left(Q^{2}\right)$, the radiated energy loss grows only logarithmically with the number, $m$, of elastic collisions in the medium. Therefore, for a random walk leading to $Q^{2} \propto m$, the induced radiation energy loss per collision, $d E / d m \propto \log (m) / m$, becomes negligible for large $m$. In the general case, between these extreme limits, there is a partial contribution from intermediate current elements. 
The essential parameter controlling this interference effect is the ratio of the formation time (11) to the distance between multiple interactions. For an ultra-relativistic particle propagating in a straight line

$$
k\left(x_{i}-x_{j}\right)=\left(\omega-k_{z}\right) L_{i j}=L_{i j} / \tau(k)
$$

where $L_{i j}=z_{i}-z_{j}$ is the longitudinal distance (time) between scattering at $i$ and $j$. For finite deflection angles, an additional phase, $-\mathbf{k}_{\perp} \cdot \mathbf{r}_{i j}$, appears that depends on the transverse separation of the scattering events. Interference between current elements $J_{i}$ and $J_{j}$ occurs only if $k\left(x_{i}-x_{j}\right) \ll 1$. This requires $\tau(k) \gg L_{i j}$ and $k_{\perp} \ll r_{i j}$. The interference pattern depends also on the current correlation function, $\left\langle J_{i}(k) J_{j}(-k)\right\rangle$. The LPM effect in QED often refers to the specific destructive interference pattern calculated by Migdal using the Fokker-Planck transport equation to solve for the probability distribution, $W\left(\mathbf{x}, \mathbf{p}, \mathbf{p}^{\prime}\right)$, of scattering points and initial and final momenta. Monte Carlo methods have also been developed [17] to calculate the development of very high energy cosmic ray air showers. The interference effect found in the limit that the scattering medium is much thicker than the radiation length is that the familiar soft $1 / \omega$ bremsstrahlung frequency spectrum is transformed into a $1 / \sqrt{ } \omega$ form for $\omega<E_{0}^{2} / E_{L P M}$. Remarkably, because the characteristic energy, $E_{L P M} \sim 3 \mathrm{TeV} \sim 5$ ergs, turns out to be so large, this interesting prediction has yet to be verified quantitatively experimentally 18.

In the following sections, we calculate the induced non-abelian radiation for a high energy parton passing through the random color field produced by a color neutral ensemble of static partons. This idealized system is chosen to minimize the complications of multiple scattering theory while illustrating the essential features of of the non-abelian LPM effect. In section 2, we first calculate the elastic multiple collision amplitude for a spinless high energy jet parton belonging to an arbitrary $S U(N)$ representation. We show how classical multiple collision cascade theory emerges from pQCD in both the high and low momentum transfer regions in the limit where the mean free path is large compared to the range of the Debye screened potentials. In section 3, we calculate the soft gluon radiation amplitudes in the restricted kinematic 
region range $k_{\perp} \ll \mu$. This restriction limits the applicability of the results to "thin" plasmas, with the number of mean free paths $m$ not large, or to very small $x \ll 1 / \sqrt{m}$ gluons. The extension of the results to thick plasmas, that would be necessary to make contact with the Migdal $\left(R=\infty, E_{0} \rightarrow \infty\right)$ limit [1], is not considered in this paper. The soft eikonal approximations used here are aimed, on the other hand, to the study of the breakdown of factorization in the opposite limit $\left(E_{0}=\infty, R \rightarrow \infty\right)$. For high energy reactions involving finite nuclei, this is in fact the only physically relevant limit for applications of pQCD in any case. Destructive interference between the radiation amplitudes from jet and exchanged gluon lines is shown to limit the transverse momentum distributions, and the non-abelian generalization of (2) is derived. In section 4, we introduce and calculate the "radiation formation factor" controlling the magnitude of the suppression of induced radiation in pQCD. We emphasize the role of color algebra on the destructive interference pattern. In section 5, the radiative energy loss, $d E / d z$, due to soft induced radiation is estimated. Finally, a discussion of remaining open problems is presented in section 6 .

\section{Multiple Elastic Scattering in a Color Neutral Ensemble}

\subsection{The Model Potential}

Consider the sequential elastic scattering of a high energy (jet) parton in the random color field produced by an ensemble of $m$ static partons located at $\mathbf{x}_{i}=\left(z_{i}, \mathbf{x}_{\perp i}\right)$ such that $z_{i+1}>z_{i}$ and $\left(z_{i+1}-z_{i}\right) \gg \mu^{-1}$, where $\mu$ is the color screening mass in the medium. As a simplified model of multiple scattering in a color neutral quark-gluon plasma, we assume a static Debye screened potential for each target parton:

$$
V_{i}^{a}(\mathbf{q})=g\left(T_{i}^{a}\right)_{c, c^{\prime}} e^{\left(-i \mathbf{q} \cdot \mathbf{x}_{i}\right)} /\left(\mathbf{q}^{2}+\mu^{2}\right)
$$

where $T_{i}^{a}$ is a $d_{i}$-dimensional generator of $S U(N)$ corresponding to the representation of the target parton at $\mathbf{x}_{i}$. The initial and final color indices, $c, c^{\prime}$, refer to the target parton are averaged and summed over when computing the ensemble averaged 
cross sections. With $V_{i}^{a} \propto T_{i}^{a}$ the ensemble averaged potential vanishes everywhere, $\left\langle V_{i}^{a}\right\rangle \propto \operatorname{Tr} T_{i}^{a}=0$. However, since

$$
\operatorname{Tr} T_{i}^{a} T_{j}^{b}=\delta_{a b} \delta_{i j}\left(d_{i} / d_{A}\right) C_{2 i}
$$

the diagonal mean square fluctuations and the cross sections are finite. Recall that for $S U(N)$ the second order Casimir, $C_{2 i}=\left(N^{2}-1\right) / 2 N \equiv C_{F}$ for quarks in the fundamental $\left(d_{i}=N\right)$ representation, while $C_{2 i}=N \equiv C_{A}$ for gluons in the adjoint $\left(d_{i}=N^{2}-1 \equiv d_{A}\right)$ representation.

In this potential, each scattering leads on the average to only a relatively small momentum transfer $q_{i}^{\mu}=\left(q_{i}^{0}, q_{z i}, \mathbf{q}_{\perp i}\right)$ with each component being much less than the incident energy, $E_{0}$. The assumption that the potentials are static is approximately valid in a high temperature plasma of massless quarks and gluons in the following sense: As $T \rightarrow \infty$, the effective coupling $g \rightarrow 0$ (albeit very slowly). The perturbative Debye screening mass $\mu \sim g T$ limits $q_{\perp} \lesssim g T$. The typical thermal energy $E_{T} \sim 3 T$ of the plasma constituents is therefore large compared to $\mu$. Consequently, the average energy loss per elastic collision, $-q^{0} \approx-q^{z} \approx q_{\perp}^{2} / 2 E_{T} \propto g^{2} T$, is $\sim g$ times smaller than the average transverse momentum transfer.

Because we are interested in relatively low momentum transfer scattering $\left(\Lambda_{Q C D} \ll\right.$ $\left.q_{\perp} \sim g T \ll T\right)$, the spin of the partons can be neglected. The jet parton is allowed, however, to be in an arbitrary $d$-dimensional representation of $S U(N)$ with generators, $T^{a}$, satisfying $T^{a} T^{a}=C_{2} \mathbf{1}_{d}$.

The Born (color matrix) amplitude to scatter from an incident four momentum $p_{i-1}^{\mu}$ to $p_{i}^{\mu}$ in the potential centered at $\mathbf{x}_{i}$ is then given by

$$
M_{i}\left(p_{i}, p_{i-1}\right)=2 \pi \delta\left(p_{i}^{0}-p_{i-1}^{0}\right) A_{i}\left(\mathbf{q}_{i}\right) e^{-i \mathbf{q}_{i} \cdot \mathbf{x}_{i}}
$$

where $\mathbf{q}_{i}=\mathbf{p}_{i}-\mathbf{p}_{i-1}$, and $A_{i}$ is shorthand for

$$
A_{i}\left(\mathbf{q}_{i}\right)=T^{a} A_{i}^{a}\left(\mathbf{q}_{i}\right)=-2 i g E_{0} T^{a} V_{i}^{a}\left(\mathbf{q}_{i}\right)
$$

The differential cross section averaged over initial and summed over final colors of both projectile and target partons reduces to the familiar form for low transverse 
momentum transfers:

$$
d \sigma_{i} / d q_{\perp i}^{2} \approx C_{i} \frac{4 \pi \alpha^{2}}{\left(q_{\perp i}^{2}+\mu^{2}\right)^{2}}
$$

where the color factor is

$$
C_{i}=\frac{1}{d d_{i}} \operatorname{Tr}\left(T^{a} T^{b}\right) \operatorname{Tr}\left(T_{i}^{a} T_{i}^{b}\right)=C_{2} C_{2 i} / d_{A}
$$

For $S U(3), 2 C_{i}$ gives the usual color factors $4 / 9,1,9 / 4$ for $q q, q g, g g$ scattering respectively. In our notation, the angular distribution is given by

$$
d \sigma_{i} / d \Omega_{i}=\frac{1}{d d_{i}} \operatorname{Tr}\left|A_{i}\left(\mathbf{q}_{i}\right)\right|^{2} /(4 \pi)^{2} .
$$

\subsection{Sequential Multiple Scattering}

Our main assumption for computing the multiple elastic scattering amplitude is that the scattering centers are well separated in the sense $L_{i}=z_{i+1}-z_{i} \gg \mu^{-1}$. In a quarkgluon plasma at very high temperature $T, 1 / \mu \sim 1 / g T$ and the effective $q g$ scattering cross section from eq.(9) is $\sigma \sim 2 \pi \alpha^{2} / \mu^{2} \sim g^{2} / 8 \pi T^{2}$. Given a Stefan-Boltzmann density of partons $\rho \sim 5 T^{3}$, the mean free path is $\lambda \sim 5 /\left(g^{2} T\right) \gg d \sim 1 / g T$ for $g \ll 1$. Hence, $L_{i} \gg d$ is satisfied at extreme temperatures at least.

The dominant Born amplitude for coincident sequential scattering with target partons from $i$ to $j$ without radiation is then simply

$$
M_{j i}\left(p_{j}, p_{i-1}\right)=\int \frac{d^{4} p_{i}}{(2 \pi)^{4}} \cdots \frac{d^{4} p_{j-1}}{(2 \pi)^{4}} M_{j}\left(p_{j}, p_{j-1}\right) i \Delta\left(p_{j-1}\right) \cdots i \Delta\left(p_{i}\right) M_{i}\left(p_{i}, p_{i-1}\right)
$$

where $\Delta(p)=\left(p^{2}-m^{2}+i \epsilon\right)^{-1}$ is the intermediate jet parton propagator. Amplitudes involving backscattering are suppressed at high energies because of the limited momentum transfer that each potential can impart. Because of the energy delta function in eq.(7), the integrations over the intermediate $p_{i}^{0}$ set all of them to $E_{0}$ and lead to a conservation factor

$$
\delta(j i) \equiv 2 \pi \delta\left(p_{j}^{0}-p_{i-1}^{0}\right) .
$$

Therefore,

$$
M_{j i}\left(p_{j}, p_{i-1}\right)=\delta(j i) \int \frac{d^{3} \mathbf{p}_{i}}{(2 \pi)^{3}} \cdots \frac{d^{3} \mathbf{p}_{j-1}}{(2 \pi)^{3}} e^{-i\left(\mathbf{p}_{j}-\mathbf{p}_{j-1}\right) \cdot \mathbf{x}_{j}} A_{j}\left(\mathbf{p}_{j}-\mathbf{p}_{j-1}\right)
$$




$$
\times\left\{\prod_{k=i}^{j-1} e^{i \pi / 2} e^{-i\left(\mathbf{p}_{k}-\mathbf{p}_{k-1}\right) \cdot \mathbf{x}_{k}} \frac{A_{k}\left(\mathbf{p}_{k}-\mathbf{p}_{k-1}\right)}{P_{0}^{2}-\mathbf{p}_{k}^{2}+i \epsilon}\right\}
$$

where $P_{0}=\left(E_{0}^{2}-m^{2}\right)^{1 / 2} \approx E_{0}$, and the product is path ordered from left to right with decreasing index $k$. Rearranging the phases in terms of the separation vectors

$$
\mathbf{R}_{k} \equiv L_{k} \hat{e}_{z}+\mathbf{r}_{k}=\mathbf{x}_{k+1}-\mathbf{x}_{k}
$$

we can write

$$
M_{j i}\left(p_{j}, p_{i-1}\right)=\delta(j i) e^{-i \mathbf{p}_{j} \cdot \mathbf{x}_{j}} e^{+i \mathbf{p}_{i-1} \cdot \mathbf{x}_{i}} I_{j i}\left(p_{j}, p_{i-1}\right),
$$

where the reduced amplitude is

$$
I_{j i}\left(p_{j}, p_{i-1}\right)=\int\left\{\prod_{k=i}^{j-1} \frac{d^{3} \mathbf{p}_{k}}{(2 \pi)^{3}} \frac{e^{i \pi / 2} e^{+i \mathbf{p}_{k} \cdot \mathbf{R}_{k}}}{P_{0}^{2}-\mathbf{p}_{k}^{2}+i \epsilon}\right\} A_{j}\left(\mathbf{p}_{j}-\mathbf{p}_{j-1}\right) \cdots A_{i}\left(\mathbf{p}_{i}-\mathbf{p}_{i-1}\right)
$$

Because of the assumed ordering, $L_{k}>0$, and the integrals over $p_{z k}$ can be evaluated by closing the contour in the upper half plane, setting the intermediate jet legs on-shell with

$$
p_{z k}=\left(P_{0}^{2}-p_{\perp k}^{2}\right)^{1 / 2} \approx P_{0}-p_{\perp k}^{2} / 2 P_{0}
$$

The singularities of the $A_{k}$ at $p_{z k} \approx P_{0}+i\left(q_{\perp k}^{2}+\mu^{2}\right)^{1 / 2}$ can be neglected because they leave very small residues $\propto \exp \left(-\mu L_{k}\right)$ given the assumed large separation $\mu L_{k} \gg 1$ between scattering centers. Therefore,

$$
I_{j i}\left(p_{j}, p_{i-1}\right) \approx \int\left\{\prod_{k=i}^{j-1} \frac{d^{2} \mathbf{p}_{\perp k}}{(2 \pi)^{2}} \frac{e^{+i \mathbf{p}_{k} \cdot \mathbf{R}_{k}}}{2 P_{0}}\right\} A_{j}\left(\mathbf{p}_{j}-\mathbf{p}_{j-1}\right) \cdots A_{i}\left(\mathbf{p}_{i}-\mathbf{p}_{i-1}\right)
$$

with $p_{z k}$ given by eq.(18). Note that in the high energy limit $I_{j i}$ survives in spite of the $1 / P_{0}$ residues because $A_{k} \propto E_{0}$ due to the vector nature of the coupling. Also the ordering of the potentials in eq.(14, 19) in decreasing order of the index cannot be permuted in the non-abelian case because of the non-commuting color matrices in the $A_{i}$. 
From eq.(19) we can derive two interesting limits. One is the semi-classical (large angle) cascade limit, and the other is the eikonal (straight line) limit for multiple small momentum transfer scatterings. The first case illustrates how the transverse momentum integrations can decouple resulting in a factorized form of the multiple collision amplitude and is discussed in Appendix A. The second limit is however physically more relevant and is considered below .

\subsection{Color Algebra}

To make explicit the color algebra we write

$$
M_{j i}=\left(a_{j} \cdots a_{i}\right) M_{j i}^{a_{j} \cdots a_{i}},
$$

in a shorthand notation where

$$
\left(a_{j} \cdots a_{i}\right) \equiv T^{a_{j}} \cdots T^{a_{i}}
$$

and we adopt the usual summation convention over repeated indices. For the color neutral ensemble under consideration

$$
M_{j i}^{a_{j} \cdots a_{i}} \propto\left(T_{j}^{a_{j}}\right)_{c_{j} c_{j}^{\prime}} \cdots\left(T_{i}^{a_{i}}\right)_{c_{i} c_{i}^{\prime}} .
$$

Hence,

$$
\left\langle M_{j i}^{a_{j} \cdots a_{i}}\left(M_{j i}^{a_{j}^{\prime} \cdots a_{i}^{\prime}}\right)^{\dagger}\right\rangle \propto \prod_{k=i}^{j} \frac{1}{d_{k}} \operatorname{Tr}\left(T_{k}^{a_{k}} T_{k}^{a_{k}^{\prime}}\right) \propto \prod_{k=i}^{j}\left(\delta_{a_{k} a_{k}^{\prime}} C_{2 k} / d_{A}\right)
$$

Given eq.(23), the color factor associated with the jet parton is given by

$$
C(i, j)=\frac{1}{d} \operatorname{Tr}\left(\left(a_{i} \cdots a_{j}\right)\left(a_{j} \cdots a_{i}\right)\right)=C_{2}^{j-i+1},
$$

as obtained by repeated use of the basic $(a a)=C_{2} \mathbf{1}_{d}$ relation. This is simply the product of the color factors, $C_{2}$, occurring for each isolated collision as in eq.(10). Therefore, even though the amplitude eq.(20) does not factor in color space, the ensemble averaged coincidence cross section does factor for large angle elastic scattering in a locally color neutral ensemble as shown in appendix A. 


\subsection{Eikonal Limit}

For a high energy jet, the coincidence amplitude is dominated by small angle scattering. In this case, we change variables to $\mathbf{q}_{l}=\mathbf{p}_{l}-\mathbf{p}_{l-1}$ with $p_{z l} \approx P_{0} \approx E_{0}$ approximately fixed, and write the total momentum transfer as

$$
\mathbf{Q}_{j i} \equiv \sum_{l=i}^{j} \mathbf{q}_{l}=\mathbf{p}_{j}-\mathbf{p}_{i-1}
$$

The coincidence amplitude can then reduces to

$$
\begin{gathered}
M_{j i}\left(p_{j}, p_{i-1}\right) \approx \delta(j i)\left(a_{j} \cdots a_{i}\right)(-i g)^{j-i+1} 2 E_{0} \int\left\{\prod_{k=i}^{j} \frac{d^{2} \mathbf{q}_{\perp k}}{(2 \pi)^{2}} e^{-i \mathbf{q}_{k} \cdot \mathbf{x}_{k}} V_{k}^{a_{k}}\left(\mathbf{q}_{k}\right)\right\} \\
\times(2 \pi)^{2} \delta^{2}\left(\mathbf{Q}_{\perp j i}-\sum_{l=i}^{j} \mathbf{q}_{\perp l}\right)
\end{gathered}
$$

Note that the dependence of the phase on the $z_{k}$ can be factored out with

$$
\sum_{k=i}^{j} q_{z k} z_{k} \approx\left(p_{z j}-P_{0}\right) z_{j}+\left(P_{0}-p_{z, i-1}\right) z_{i} .
$$

This phase is important only for off-shell amplitudes with $p_{z j} \neq P_{0}$ or $p_{z, i-1} \neq P_{0}$.

To average over the transverse coordinates $\mathbf{x}_{k}$, we employ the frozen target approximation taking the initial and final wavefunction of target parton, $k$, to be $\phi_{k i}\left(\mathbf{x}_{k}\right)$ and by $\phi_{k f}\left(\mathbf{x}_{k}\right)$ respectively. The amplitude to leave the target in a specific final state is obtained by replacing the phase factors by transition form factors

$$
e^{-i \mathbf{q}_{k} \cdot \mathbf{x}_{k}} \rightarrow F_{i f}^{k}\left(\mathbf{q}_{k}\right)=\int d^{3} x_{k} \phi_{k f}^{*}\left(\mathbf{x}_{k}\right) e^{-i \mathbf{q}_{k} \cdot \mathbf{x}_{k}} \phi_{k i}\left(\mathbf{x}_{k}\right)
$$

After squaring $M_{j i}$, we must sum over all final states $\phi_{k f}$. For scattering in a chaotic or thermal bath we must also average over an ensemble of initial $\phi_{k i}$.

A simplification is possible in the high energy limit when the energy and longitudinal momentum transfers are small, and they can be neglected or replaced by their average values in $\delta(j i)$ and $F_{i f}^{k}$. In that case, closure $\left(\sum_{f} \phi_{k f} \phi_{k f}^{*}=1\right)$ can be applied 
to the sum over final states. Averaging in addition over initial states with probability $p(i)$, the average squared amplitude contains factors such as

$$
\sum_{i} p(i) \sum_{f} F_{i f}^{k}\left(\mathbf{q}_{\perp k}\right) F_{f i}^{k}\left(-\mathbf{q}_{\perp k}^{\prime}\right)=\int d^{3} x_{k} e^{-i\left(\mathbf{q}_{\perp k}-\mathbf{q}_{\perp k}^{\prime}\right) \cdot \mathbf{x}_{k}} \rho_{k}\left(\mathbf{x}_{k}\right) \equiv T_{k}\left(\mathbf{q}_{\perp k}-\mathbf{q}_{\perp k}^{\prime}\right)
$$

where $\rho_{k}\left(\mathbf{x}_{k}\right)=\sum_{i} p(i)\left|\phi_{k 0}\left(\mathbf{x}_{k}\right)\right|^{2}$ is the ensemble average density distribution of target parton $k$. In eq.(29) note that $T_{k}\left(\mathbf{q}_{\perp}\right)$ is just the Fourier transform of the Glauber thickness function

$$
T_{k}\left(\mathbf{x}_{\perp k}\right)=\int d z \rho_{k}\left(z, \mathbf{x}_{\perp k}\right)
$$

which is the probability per unit area of finding parton $k$ at a transverse coordinate $\mathbf{x}_{\perp k}$. For a broad $z$ distribution, it would appear that we may have violated the assumed $\mu L \gg 1$ assumption. However, in the $m$ ! different $z$ orderings of the centers, one of the previously neglected backscattering amplitudes becomes dominant and after relabelling the dummy indices the same result is recovered. The only essential assumption is that the mean free path is long compared to the range of the potential.

With eq.(29), the ensemble average of the squared amplitude is proportional to

$$
\begin{aligned}
\left\langle\left|M_{j i}\left(p_{i-1}, p_{j}\right)\right|^{2}\right\rangle \propto \int & \left\{\prod_{k=i}^{j} \frac{d^{2} \mathbf{q}_{\perp k}}{(2 \pi)^{2}} \frac{d^{2} \mathbf{q}_{\perp k}^{\prime}}{(2 \pi)^{2}} T_{k}\left(\mathbf{q}_{\perp k}-\mathbf{q}_{\perp k}^{\prime}\right) V_{k}^{a_{k}}\left(\mathbf{q}_{k}\right)\left(V_{k}^{a_{k}}\left(\mathbf{q}_{k}^{\prime}\right)\right)^{*}\right\} \\
& \times(2 \pi)^{4} \delta^{2}\left(\mathbf{Q}_{\perp j i}-\sum_{l=i}^{j} \mathbf{q}_{\perp l}\right) \delta^{2}\left(\mathbf{Q}_{\perp j i}-\sum_{l=i}^{j} \mathbf{q}_{\perp l}^{\prime}\right) .
\end{aligned}
$$

Only diagonal color components survive because of the color neutrality condition eq.(23). If the transverse coordinates are distributed over a radius, $R \gg \mu^{-1}$, then the Fourier transform of the thickness function will limit the difference, $\left|\mathbf{q}_{\perp k}-\mathbf{q}_{\perp k}^{\prime}\right| \lesssim 1 / R$. Because $V_{k}^{a_{k}}\left(\mathbf{q}_{k}\right)$ varies slowly on a scale $1 / R \ll \mu$, we can therefore approximate $\left(V_{k}^{a_{k}}\left(\mathbf{q}_{k}^{\prime}\right)\right)^{*} \approx\left(V_{k}^{a_{k}}\left(\mathbf{q}_{k}\right)\right)^{*}$ in the integrand. The $\mathbf{q}_{k}^{\prime}$ integrals result therefore in a 
multiplicative geometrical factor

$$
\int\left\{\prod_{k=i}^{j} \frac{d^{2} \mathbf{q}_{\perp k}^{\prime}}{(2 \pi)^{2}} T_{k}\left(\mathbf{q}_{\perp k}-\mathbf{q}_{\perp k}^{\prime}\right)\right\}(2 \pi)^{2} \delta^{2}\left(\sum_{l=i}^{j}\left(\mathbf{q}_{\perp l}-\mathbf{q}_{\perp l}^{\prime}\right)\right)=\int d^{2} \mathbf{b} \prod_{k=i}^{j} T_{k}(\mathbf{b})
$$

With the above simplification, the ensemble averaged coincidence cross section to scatter sequentially with partons from $i$ to $j$ reduces to

$$
d \sigma_{j i} / d^{2} \mathbf{Q}_{\perp j i}=\int d^{2} \mathbf{b} \int \prod_{k=i}^{j}\left\{d^{2} \mathbf{q}_{\perp k} T_{k}(\mathbf{b}) d \sigma_{k} / d^{2} \mathbf{q}_{\perp k}\right\} \delta^{2}\left(\mathbf{Q}_{\perp j i}-\sum_{l=i}^{j} \mathbf{q}_{\perp l}\right)
$$

This is recognized as the classical Glauber multiple collision limit, with $p_{k}(\mathbf{b})=$ $\int T_{k}(\mathbf{b}) d \sigma_{k}$ being the probability of scattering off center $k$ on a classical trajectory at impact parameter $\mathbf{b}$.

\section{Induced Soft Non-Abelian Radiation}

We turn next to the inelastic amplitudes for induced radiation of a gluon with color $c$ and light cone momenta and polarization

$$
\begin{aligned}
k^{\mu} & =\left(\omega, k_{z}, \mathbf{k}_{\perp}\right)=\left[x P^{+}, k_{\perp}^{2} / x P^{+}, \mathbf{k}_{\perp}\right] \\
\epsilon^{\mu} & =\left(\epsilon_{0},-\epsilon_{0}, \vec{\epsilon}_{\perp}\right)=\left[0,2 \vec{\epsilon}_{\perp} \cdot \mathbf{k}_{\perp} / x P^{+}, \vec{\epsilon}_{\perp}\right] .
\end{aligned}
$$

Light cone coordinates are denoted here by square brackets, $\left[k^{+}, k^{-}, \mathbf{k}_{\perp}\right]$, with $k^{ \pm}=$ $\omega \pm k_{z}=k_{\perp}^{2} / k^{\mp}$. We chose the two physical polarization states for on shell $\left(k^{2}=0\right)$ gluons to satisfy both $\epsilon k=0$ and $\epsilon n=0$ with $n^{\mu}=\left[0,2,0_{\perp}\right]$ in terms of two orthonormal $\vec{\epsilon}_{\perp}$. Thus $\epsilon^{0}=\vec{\epsilon}_{\perp} \cdot \mathbf{k}_{\perp} /\left(\omega+k_{z}\right)$. In light cone coordinates, the incident jet parton has $p_{0}^{\mu}=\left[P^{+}, m^{2} / P^{+}, 0\right]$ with $P^{+} \approx 2 E_{0}$. We focus on the soft limit defined by $x \ll 1$. First we consider the amplitudes for radiation from the high energy parton lines. Then we show that the three gluon amplitudes essentially cut off the soft $d k_{\perp} / k_{\perp}$ spectrum at $k_{\perp} \sim \mu$. Note that the induced bremsstrahlung associated with a single isolated collision was derived in pQCD in Ref. 19. Our interest here is on the induced radiation pattern associated with multiple collisions. 


\subsection{Radiation from Internal Jet lines}

The amplitude to emit a gluon with color $c$ from the $j$ th intermediate jet line during sequential scattering with target partons from 1 to $m$ is

$$
\begin{aligned}
M_{m 1}^{c j}\left(k, p_{m}, p_{0}\right)= & \int \frac{d^{4} p_{j}}{(2 \pi)^{4}} M_{m, j+1}\left(p_{m}, p_{j}-k\right) \\
& \times\left\{i \Delta\left(p_{j}-k\right)\left(-2 i g \epsilon p_{j} T^{c}\right) i \Delta\left(p_{j}\right)\right\} M_{j, 1}\left(p_{j}, p_{0}\right)
\end{aligned}
$$

The integrals over $p_{j}^{0}$ set their values to $E_{0}$ because of the $\delta(1 j)$ in $M_{j, 1}$. However, for all the subsequent intermediate lines in $M_{m, j+1}$ the energy is shifted from $E_{0}$ to $E_{0}-\omega$, and a new overall energy conservation factor arises

$$
\tilde{\delta}(m 1) \propto \delta\left(p_{m}^{0}-E_{0}+\omega\right)
$$

These shifted energies change the classical momenta in subsequent legs $(k>j)$ to $\mathbf{P}_{k}=P_{\omega} \hat{\mathbf{R}}_{k}$ with

$$
P_{\omega}=\left(\left(E_{0}-\omega\right)^{2}-m^{2}\right)^{1 / 2} \approx P_{0}-\omega / v_{0}
$$

where $v_{0}=P_{0} / E_{0}$ is the speed of the incident parton.

To perform the $p_{z j}$ integral, it is convenient to split the two propagators using

$$
2 \epsilon p_{j} \Delta\left(p_{j}-k\right) \Delta\left(p_{j}\right)=\frac{\epsilon\left(p_{j}-k\right)}{k\left(p_{j}-k\right)} \Delta\left(p_{j}-k\right)-\frac{\epsilon p_{j}}{k_{\mu} p_{j}^{\mu}} \Delta\left(p_{j}\right),
$$

which is valid for on shell radiation since $k^{2}=0$ and $\epsilon k=0$. The contour integral over $p_{z j}$ can then be performed as discussed in Appendix B.

With (123,124) from Appendix B, the radiation amplitude eq.(35) reduces in the kinematic region $x \ll 1$ and $k_{\perp} \ll \mu$ to

$$
\begin{aligned}
M_{m 1}^{c j} \approx g \tilde{\delta} & m 1) e^{-i \omega t_{m}} e^{-i \mathbf{p}_{m} \cdot \mathbf{x}_{m}} e^{+i \mathbf{p}_{0} \cdot \mathbf{x}_{1}} \int \frac{d^{2} \mathbf{p}_{\perp j}}{(2 \pi)^{2}} \frac{1}{2 P_{0}} e^{i\left(\mathbf{P}_{j} \cdot \mathbf{R}_{j}-\frac{L_{j}}{2 P_{j}} \mathbf{p}_{\perp j}^{2}\right)} \\
& \times\left(\frac{\epsilon \tilde{p}_{j}}{k \tilde{p}_{j}} e^{+i k x_{j}}-\frac{\epsilon p_{j}}{k p_{j}} e^{+i k x_{j+1}}\right) I_{m, j+1}\left(p_{m}, p_{j}\right) T^{c} I_{j, 1}\left(p_{j}, p_{0}\right)
\end{aligned}
$$

Note the appearance of the phases $k x_{j}$ and $k x_{j+1}$ where $x_{j}^{\mu}=\left(t_{j}, \mathbf{x}_{j}\right)$ and the interaction time, $t_{j}$, is the classical transit time along the path from $\mathbf{x}_{1}$ to $\mathbf{x}_{j}$ as defined in 
eq.(121). In addition, the current element in the brackets involves on-shell momenta, $p_{j}^{\mu}, \tilde{p}_{j}^{\mu}$, defined by

$$
\begin{gathered}
p_{j}^{\mu}=\left(E_{0},\left(P_{0}^{2}-\left(\mathbf{P}_{\perp j}+\mathbf{p}_{\perp j}\right)^{2}\right)^{1 / 2}, \mathbf{P}_{\perp j}+\mathbf{p}_{\perp j}\right) \\
\tilde{p}_{j}^{\mu}=\left(E_{0}-\omega,\left(P_{\omega}^{2}-\left(\mathbf{P}_{\perp j}+\mathbf{p}_{\perp j}\right)^{2}\right)^{1 / 2}, \mathbf{P}_{\perp j}+\mathbf{p}_{\perp j}\right) .
\end{gathered}
$$

Note finally the order in which the color matrix $T^{c}$ appears above.

For radiation with $k_{\perp} \ll \mu$, we can factor out of the integrand a current element proportional to $\epsilon P_{j} / k P_{j}$. To see this, note first that for a high energy on-shell parton with $p=\left[(1-\delta) P^{+}, m_{\perp}^{2} /(1-\delta) P^{+}, \mathbf{p}_{\perp}\right]$ and radiation with kinematics (34),

$$
\frac{\epsilon p}{k p}=2 \frac{\vec{\epsilon}_{\perp} \cdot\left(\mathbf{k}_{\perp}-x \mathbf{p}_{\perp} /(1-\delta)\right)}{\left(\mathbf{k}_{\perp}-x \mathbf{p}_{\perp} /(1-\delta)\right)^{2}+x^{2} m^{2} /(1-\delta)^{2}} \approx 2 \frac{\vec{\epsilon}_{\perp} \cdot \mathbf{k}_{\perp}}{k_{\perp}^{2}} \quad \text { for } x m_{\perp} \ll k_{\perp} .
$$

This approximate independence of the current element on $p^{\mu}$ allows us to factor out (42) from the integrals for $x m_{\perp} \ll k_{\perp}$. However, a more general expression can be factored out that is valid also in the high momentum transfer limit. For fixed $\mathbf{x}_{i}$ and $E_{0} \rightarrow \infty$, the momenta $p_{j}^{\mu}$ and $\tilde{p}_{j}^{\mu}$ are approximately fixed by geometry to be $P_{j}^{\mu} \approx E_{0}\left(1, \hat{\mathbf{R}}_{j}\right)$, and therefore

$$
\frac{\epsilon p_{j}}{k p_{j}} \approx \frac{\epsilon \tilde{p}_{j}}{k \tilde{p}_{j}} \approx \frac{\epsilon P_{j}}{k P_{j}}
$$

Since this expression also reduces to (42) when $\hat{\mathbf{R}}_{j}$ points along the jet direction, the final factorized form of the amplitude for intermediate line radiation becomes

$$
M_{m 1}^{c j} \approx g \frac{\epsilon P_{j}}{k P_{j}}\left(e^{i k x_{j}}-e^{i k x_{j+1}}\right)\left(a_{m} \cdots a_{j+1} c a_{j} \cdots a_{1}\right) M_{m 1}^{a_{m} \cdots a_{1}}
$$

In this expression, the $j$ independent phase factor, $\exp \left(-i \omega t_{m}\right)$, was discarded.

We note several points in connection the amplitudes for induced radiation from the internal jet lines given by eq.(44).

1. The approximate factored expression holds both in the large angle cascade limit considered in Appendix A and straight line (Eikonal) limits of $M_{m 1}$ as long as $x \ll 1$ and $k_{\perp} \ll \mu$. 
2. Just as in the QED case, these intermediate line amplitudes vanish for radiation with formation length significantly exceeding the separation of adjacent scattering centers. In particular, for fixed $x=k^{+} / P^{+} \approx \omega / E_{0} \ll 1$ the phase factors cancel in the $k_{\perp} \rightarrow 0$ limit:

$M_{m 1}^{c j} \propto k\left(x_{j+1}-x_{j}\right)=\omega L_{j}\left(1 / v_{0}-\cos \theta\right)-\mathbf{k}_{\perp} \cdot \mathbf{r}_{\perp j} \approx L_{j} / \tau(k)-\mathbf{k}_{\perp} \cdot \mathbf{r}_{\perp j} \rightarrow 0$

where $\tau(k)$ is the formation time (length) from eq.(11).

3. Unlike in momentum space, there is no factorization in color space. The color matrix for the amplitude without radiation is modified by radiating a gluon of color $c$ after the $j$ th interaction by the insertion of a $T^{c}$ matrix:

$$
\left(a_{m} \cdots a_{j} \cdots a_{1}\right) \rightarrow\left(a_{m} \cdots c a_{j} \cdots a_{1}\right)
$$

4. Even for $x \ll 1$ the condition leading to the approximate form in (42) breaks down for very large number of collisions since the random walk in transverse momentum space leads to a growing $\left\langle p_{\perp m}^{2}\right\rangle \propto m \mu^{2}$. Thus for large $m$ the above approximation is only valid in a restricted $x$ region

$$
x \ll \frac{1}{\sqrt{ } m} \frac{k_{\perp}}{\mu} \ll \frac{1}{\sqrt{ } m} .
$$

5. We must also add the amplitudes for radiation from the initial and final lines to the above amplitudes for radiation from internal lines. These external line amplitudes are

$$
\begin{aligned}
M_{m 1}^{c 0} & =M_{m 1}\left(p_{m}, p_{0}-k\right)\left(i \Delta\left(p_{0}-k\right)\right)\left(-2 i g \epsilon p_{0} T^{c}\right) \\
& \approx-g \frac{\epsilon P_{0}}{k P_{0}} e^{i k x_{1}}\left(a_{m} \cdots a_{1} c\right) M_{m 1}^{a_{m} \cdots a_{1}} \\
M_{m 1}^{c m} & =\left(i \Delta\left(p_{m}\right)\right)\left(-2 i g \epsilon p_{m} T^{c}\right) M_{m 1}\left(p_{m}, p_{0}\right) \\
& \approx g \frac{\epsilon P_{m}}{k P_{m}} e^{i k x_{m}}\left(c a_{m} \cdots a_{1}\right) M_{m 1}^{a_{m} \cdots a_{1}}
\end{aligned}
$$

where we defined $P_{0}^{\mu}=p_{0}^{\mu}, P_{m}^{\mu}=p_{m}^{\mu}$ for notational convenience, and we again discarded the common phase factor, $\exp \left(-i \omega t_{m}\right)$, as in eq.(44). 
The sum of all the amplitudes (44,18) can then be written in a suggestive form

$$
M_{m}^{c}\left(k, p_{m}, p_{0}\right) \approx-i[\epsilon J(k)]_{a_{m} \cdots a_{1}}^{c} M_{m 1}^{a_{m} \cdots a_{1}}\left(p_{m}, p_{0}\right)
$$

where the effective color current element analogous to (2) is

$$
[\epsilon J(k)]_{a_{m} \cdots a_{1}}^{c}=i g \sum_{l=1}^{m} e^{i k x_{l}}\left(\frac{\epsilon P_{l}}{k P_{l}}\left(a_{m} \cdots c a_{l} \cdots a_{1}\right)-\frac{\epsilon P_{l-1}}{k P_{l-1}}\left(a_{m} \cdots a_{l} c \cdots a_{1}\right)\right),
$$

In the abelian case, the matrices in $(\cdots)$ are set to unity, and Eqs.(49,50) reduce to the soft radiation formulas of QED. In the abelian case the current actually vanishes as a power of $x$ in the $x \rightarrow 0$ limit on account of (42). In the non-abelian case, the non-commutativity of the generators leads, however, to a non-vanishing current in (50) even for $x=0$.

We emphasize that the approximate form of the effective current in eq.(50) is valid only in a restricted kinematic domain (47). Gauge invariance requires the absence of induced radiation associated with collisions without momentum transfer [19], i.e., for $P_{l}=P_{l-1}$. On the other hand, the contribution from scattering at $l$ in eq.(50) is nonvanishing if $[c, a] \neq 0$. To recover full gauge invariance, of course all the amplitudes involving three and four gluon vertices as well must be added to the above result. However, since the domain of applicability of eq.(50) shrinks to zero as $q_{\perp j} \rightarrow 0$, it is consistent with the gauge invariance requirement in the kinematic domain indicated.

For the physically most interesting eikonal limit, the effective color current reduces for $x \sqrt{ } m \ll k_{\perp} / \mu \ll 1$ to

$$
[\epsilon J(k)]_{a_{m} \cdots a_{1}}^{c} \approx 2 i g \frac{\vec{\epsilon}_{\perp} \cdot \mathbf{k}_{\perp}}{k_{\perp}^{2}} \sum_{l=1}^{m} e^{i k x_{l}}\left(a_{m} \cdots\left[c, a_{l}\right] \cdots a_{1}\right) .
$$

The lower bound on the domain of applicability comes from (47). For $k_{\perp}<x \mu \sqrt{ } j$, the effective current has components in the directions $\mathbf{p}_{\perp j}$ that cannot be factorized out of the elastic amplitude. We note that (51) can also be derived directly from (35) using the eikonal form (26) for $M_{m, j+1}$ and $M_{j, 1}$. The above derivation has the advantage that the connection to the familiar abelian case is made more transparent. 


\subsection{Radiation from Internal Gluon Lines}

In the previous section we concentrated on the amplitudes for induced radiation in which the gluon is radiated directly from the fast jet lines without final state interactions. Here we consider amplitudes involving one or more three or four gluon vertices in which the gluon scatters with one or more of the target partons before emerging with kinematics given by (34). In the high energy limit, the amplitudes involving the four gluon vertices can be generally neglected because those vertices are momentum independent and a contact interaction with two widely separated target partons is small. The amplitudes involving three gluon vertices can be classified by the number of such vertices and the indices of the scattering centers to which one of the gluon legs is attached. The simplest of such amplitudes, denoted by $G_{j m}^{c}$, corresponds to a (possibly virtual) gluon emitted by the jet between the $z_{j-1}$ and $z_{j+1}$ with that gluon scattering off the target parton at $z_{j}$. For $1<j<m$,

$$
G_{m j}^{c}=\int \frac{d p_{j}^{4}}{(2 \pi)^{4}} \frac{d p_{j-1}^{4}}{(2 \pi)^{4}} M_{m, j+1}\left(p_{m}, p_{j}\right) i \Delta\left(p_{j}\right) G_{j}^{c}\left(k, p_{j}, p_{j-1}\right) i \Delta\left(p_{j-1}\right) M_{j-1,1}\left(p_{j-1}, p_{0}\right)
$$

where with $q_{j}=p_{j}-p_{j-1}$ the single three gluon amplitude in the Feynman gauge is

$$
\begin{aligned}
G_{j}^{c}\left(k, p_{j}, p_{j-1}\right)=( & \left.-i g T_{b}\left(p_{j-1}+p_{j}\right)^{\alpha}\right)\left(-i \Delta\left(q_{j}\right)\right) \\
& \left.\times\left(-g f_{b a_{j} c} \Lambda_{\alpha \beta \gamma}\left(q_{j},-q_{j}-k, k\right)\right) A_{j}^{a_{j} \beta}\left(q_{j}+k\right)\right) \epsilon^{\gamma}(k) .
\end{aligned}
$$

The external field at $j$ in our case is

$$
A_{j}^{a_{j} \beta}(q)=g^{\beta 0} 2 \pi \delta\left(q^{0}\right) V_{j}^{a_{j}}(\mathbf{q}) e^{-i \mathbf{q} \cdot \mathbf{x}_{j}},
$$

and the three gluon tensor is

$$
\Lambda^{\alpha \beta \gamma}\left(p_{1}, p_{2}, p_{3}\right)=\left(p_{2}-p_{3}\right)^{\alpha} g^{\beta \gamma}+\left(p_{3}-p_{1}\right)^{\beta} g^{\gamma \alpha}+\left(p_{1}-p_{2}\right)^{\gamma} g^{\alpha \beta}
$$

The amplitudes with multiple three gluon vertices correspond to multiple final state interactions of the emitted gluon. Because the centers are assumed to be far apart, the intermediate gluon lines in those amplitudes are set on shell (by the corresponding $d p_{z}$ contour integral). Those amplitudes therefore describe final state 
cascading of the gluon in the medium. In QED these type of amplitudes are replaced by higher order Compton like amplitudes. For gluons, final state cascading will broaden their final $k_{\perp}$ distribution and induce further gluon showering. However, for the problem of the energy loss of the incident jet, which is our primary interest here, the final transverse momentum distribution of the rescattered gluons is not important. In addition, in the soft limit $k_{\perp} \ll \mu$ each triple gluon vertex gives rise to a factor $O\left(k_{\perp} / \mu\right)$ smaller than the corresponding internal jet line radiation amplitude, as we show below. We therefore concentrate here only on the amplitudes involving one three gluon vertex given by (52).

As these amplitudes only arise in the non-abelian case, we can simplify the derivation by evaluating (52) in the eikonal limit. As shown in Appendix C, we find in this limit that

$$
\begin{aligned}
G_{j m}^{c} \approx & 2 \pi \delta\left(p_{m}^{0}-E_{0}+\omega\right)(-i g)^{m}\left(2 E_{0}\right) \int \prod_{k=1}^{m}\left(\frac{d^{2} q_{\perp k}}{(2 \pi)^{2}} e^{-i \mathbf{q}_{\perp k} \cdot \mathbf{x}_{\perp k}} V_{k}^{a_{k}}\left(\mathbf{q}_{\perp k}\right)\right) \\
& \times(2 \pi)^{2} \delta\left(\mathbf{Q}_{\perp}-\mathbf{k}_{\perp}-\sum_{k=1}^{m} \mathbf{q}_{\perp k}\right)\left\{2 g e^{i z_{j} / \tau(k)} \frac{\vec{\epsilon}_{\perp} \cdot\left(\mathbf{q}_{\perp j}-\mathbf{k}_{\perp}\right)}{\left(\mathbf{q}_{\perp j}-\mathbf{k}_{\perp}\right)^{2}}\left(a_{m} \cdots\left[c, a_{j}\right] \cdots a_{1}\right)\right\}
\end{aligned}
$$

We note that corrections to the effective current element in the \{\} brackets arise for large $j$ in the region $k_{\perp}<x p_{\perp j} \sim x \mu \sqrt{ } j$ from terms neglected in the vertex function in eq. (133) of Appendix C. In eq.(56), $\mathbf{Q}_{\perp}=\mathbf{p}_{\perp m}$ is the final transverse momentum of the jet parton.

Summing these three gluon amplitudes and adding the radiation amplitudes from the jet lines in Eqs.(49,51), we obtain the total amplitude for $m$-fold coincidence scatterings together with soft radiation in the eikonal limit:

$$
\begin{aligned}
& M_{m}^{c}\left(k, p_{m}, p_{0}\right) \approx 2 \pi \delta\left(p_{m}^{0}-E_{0}+\omega\right)(-i g)^{m}\left(2 E_{0}\right) \int \prod_{k=1}^{m}\left(\frac{d^{2} q_{\perp k}}{(2 \pi)^{2}} e^{-i \mathbf{q}_{\perp k} \cdot \mathbf{x}_{\perp k}} V_{k}^{a_{k}}\left(\mathbf{q}_{\perp k}\right)\right) \\
& \times(2 \pi)^{2} \delta\left(\mathbf{Q}_{\perp}-\mathbf{k}_{\perp}-\sum_{k=1}^{m} \mathbf{q}_{\perp k}\right)\left\{-i \vec{\epsilon}_{\perp} \cdot \vec{J}_{a_{1} \cdots a_{m}}^{c}\left(k ;\left\{\mathbf{q}_{\perp i}\right\}\right)\right\}
\end{aligned}
$$


where the effective color current is

$$
\vec{J}_{a_{1} \cdots a_{m}}^{c}\left(k ;\left\{\mathbf{q}_{\perp i}\right\}\right)=\sum_{i=1}^{m} e^{i z_{i} / \tau(k)} \vec{\jmath}_{i}(k)\left(a_{m} \cdots\left[c, a_{i}\right] \cdots a_{1}\right),
$$

and the elementary current elements are

$$
\vec{\jmath}_{i}(k)=2 i g\left(\frac{\mathbf{k}_{\perp}}{k_{\perp}^{2}}+\frac{\mathbf{q}_{\perp i}-\mathbf{k}_{\perp}}{\left|\mathbf{q}_{\perp i}-\mathbf{k}_{\perp}\right|^{2}}\right) .
$$

The following points should be noted in connection with the above results:

1. For the case $m=1$, eq.(58) reduces to the result derived in [19].

2. For $k_{\perp} \ll q_{\perp i}$, the three gluon amplitudes can be neglected as noted before. Therefore, in this limit eq.(58) reduces to eq.(51).

3. However, for very small $k_{\perp}<x \mu_{\perp i}$ corrections to eq.(59) arise as can be seen from eq.(42). In particular, the singularities at $k_{\perp}=0$ is regulated on a scale $x \mu$, where $\mu$ is the (dynamic) mass of the jet parton.

4. The singularity at $k_{\perp}=q_{\perp i}$ is a non-abelian feature due to induced radiation along the direction of the exchanged gluon. It is regulated by the gluon polarization tensor in the medium 20.

5. The approximate color current is strictly valid only for $k_{\perp} \ll \mu$ and $x \ll 1$. However, eq.(59) shows the general cancellation of amplitudes for $k_{\perp} \gg q_{\perp i}$ that limits the induced radiation from an isolated scattering to $k_{\perp} \lesssim \mu$.

6. For $q_{\perp i}=0$, the current element $\vec{\jmath}_{i}$ vanishes in accordance with gauge invariance 19.

7. The phase factor in eq.(58) is independent of the transverse coordinates $\mathbf{x}_{\perp i}$ in the eikonal limit. The transverse phase factors, $\exp \left(-i \mathbf{k}_{\perp} \cdot \mathbf{x}_{\perp i}\right)$, associated with each isolated collision are spread over the net elastic phase factor $\exp \left(-i \sum_{k} \mathbf{q}_{\perp k}\right.$. $\left.\mathbf{x}_{\perp k}\right)$. 
8. Finally, corrections to eq.(59) also appear in kinematic domains where either the radiation formation time, $\tau(k)$, or the intermediate jet lifetime, $2 \omega /\left(\mathbf{q}_{\perp i}-\mathbf{k}_{\perp}\right)^{2}$, is on the order of the mean free path due as shown by eq.(137) in Appendix C.

Eqs.(58,59) are the main result of this paper from which we derive next the nonabelian LPM interference effect in the eikonal limit.

\section{The Radiation Formation Factor}

The spectrum of soft induced bremsstrahlung associated with multiple scattering in a color neutral ensemble eq.(23) can be computed from eq.(58) using steps similar to those leading from eq.(26) to eq.(33). Analogous to eq.(3), we find that

$$
\omega \frac{d^{3} n_{m}}{d^{3} k}=\frac{1}{2(2 \pi)^{3}} \frac{1}{C_{2}^{m} d}\left\langle\operatorname{Tr} \sum_{\vec{\epsilon}_{\perp}}\left|\left[\epsilon J\left(k,\left\{\mathbf{q}_{\perp i}\right\}\right)\right]_{a_{1} \cdots a_{m}}^{c}\right|^{2}\right\rangle .
$$

Recall that $C_{2}^{m}$ is the color factor for the coincidence scattering cross section without radiation from eq.(24) with $C_{2}$ and $d$ being the second order Casimir and dimension of the $S U(N)$ representation of the jet parton. As in eq.(31) the assumptions of color neutrality and that the transverse distribution of target partons is much wider than $\mu^{-1}$ are essential to obtain the above diagonal form in color and $\mathbf{q}_{\perp i}$ labels. Note that the squared current involves a sum over repeated color indices, $c, a_{i}$, and the trace is over the resulting sum of products of color matrices that we consider in detail below. The average, denoted by large brackets, above is over the transverse momentum transfers, $\mathbf{q}_{\perp i}$, and given in our case by

$$
\left\langle f\left(\mathbf{q}_{\perp i}\right)\right\rangle=\int\left\{\prod_{i=1}^{m} \frac{\mu^{2} d^{2} \mathbf{q}_{\perp i}}{\pi\left(q_{\perp i}^{2}+\mu^{2}\right)^{2}}\right\} f\left(\mathbf{q}_{\perp i}\right) .
$$

We are mainly interested in comparing the induced spectrum for $m>1$ to the radiation spectrum from a single isolated collision 19]:

$$
\omega \frac{d^{3} n_{1}}{d^{3} k}=\left\langle\frac{\alpha_{s} C_{A}}{\pi^{2}} \frac{q_{\perp}^{2}}{k_{\perp}^{2}\left(\mathbf{k}_{\perp}-\mathbf{q}_{\perp}\right)^{2}}\right\rangle_{+} .
$$


The average in this case is over a single scattering as obtained from eq.(61) by setting $m=1$. The + label on the average indicates that the infrared singularities are regulated in a quark-gluon plasma by the dynamically generated masses of the initial jet and radiated gluon as discussed in the previous section. Note that this mechanism is different from the regulation of infrared singularities considered in Ref. [19] due to form factors arising in collisions of color singlet hadrons. In that case, interference amplitudes associated with radiation from different hadronic constituents cancel both the $k_{\perp}=0$ and the $\mathbf{k}_{\perp}=\mathbf{q}_{\perp}$ singularities. In a quark-gluon plasma at the perturbative level the quasi-particles are color non-singlet partons, and the singularities are regulated by medium polarization effects [20]. Nevertheless, in both cases similar expressions arise at the end.

With eqs.(60,62), we can define the "radiation or color formation factor", $C_{m}(k)$ via

$$
\frac{d^{3} n_{m}}{d^{3} k} \equiv C_{m}(k) \frac{d^{3} n_{1}}{d^{3} k}
$$

The result can be expressed as

$$
C_{m}(k)=\frac{1}{C_{2}^{m} C_{A} d} \sum_{i=1}^{m}\left(C_{i i}+2 R e \sum_{j=1}^{i-1} C_{i j} e^{i\left(z_{i}-z_{j}\right) / \tau(k)} F_{i j}(k)\right)
$$

in terms of color coefficients,

$$
C_{i j}=\operatorname{Tr}\left(a_{m} \cdots\left[c, a_{i}\right] \cdots a_{1} a_{1} \cdots\left[a_{j}, c\right] \cdots a_{m}\right)
$$

and current correlation functions

$$
F_{i j}(k)=\left\langle\vec{\jmath}_{i} \cdot \vec{\jmath}_{j}\right\rangle /\left\langle\left|\vec{\jmath}_{i}\right|^{2}\right\rangle
$$

with $\vec{\jmath}_{i}$ given by eq. (59).

\subsection{Color Coefficients}

The color coefficients in eq.(65) can be computed by repeated use of basic $S U(N)$ relations for sums of products of generators:

$$
a a=C_{2} \mathbf{1}_{d},[a, b] a=-\frac{C_{A}}{2} b
$$




$$
a b a=\left(C_{2}-C_{A} / 2\right) b,[a, b][b, a]=C_{A} C_{2} \mathbf{1}_{d}
$$

The diagonal coefficients can be seen to be identical to the normalization factor in eq. (64):

$$
C_{i i}=\operatorname{Tr}\left(C_{2}^{m-i}\left[c, a_{i}\right] C_{2}^{i-1}\left[a_{i}, c\right]\right)=C_{2}^{m} C_{A} d
$$

The off diagonal $j<i$ coefficients are, on the other hand,

$$
\begin{aligned}
C_{i j} & =\operatorname{Tr}\left(C_{2}^{m-i}\left[c, a_{i}\right] a_{i-1} \cdots a_{j} C_{2}^{j-1}\left[a_{j}, c\right] a_{j+1} \cdots a_{i}\right) \\
& =-\frac{C_{A}^{2}}{4} C_{2}^{m-i+j-1}\left(C_{2}-\frac{C_{A}}{2}\right)^{i-j-1} C_{2} d \\
& =-r_{2}\left(1-r_{2}\right)^{i-j-1} C_{i i} / 2
\end{aligned}
$$

where

$$
r_{2}=\frac{C_{A}}{2 C_{2}}=\left\{\begin{array}{ll}
N^{2} /\left(N^{2}-1\right) & \text { for quarks with } C_{2}=C_{F} \\
1 / 2 & \text { for gluons with } C_{2}=C_{A}
\end{array} .\right.
$$

The radiation formation factor is therefore

$$
C_{m}(k)=m-\frac{r_{2}}{1-r_{2}} R e \sum_{i=1}^{m} \sum_{j=1}^{i-1}\left(1-r_{2}\right)^{i-j} F_{i j}(k) e^{i L_{i j} / \tau(k)} .
$$

For a single isolated scattering, of course,

$$
C_{1}(k)=1
$$

For multiple scattering in the Bethe-Heitler limit, corresponding to $L_{i j} \gg \tau(k)$, the phase factors average to zero, and the intensity of induced radiation is simply additive in the number of scatterings, i.e.,

$$
C_{m}(k) \approx m \text { if } L_{i j} \gg \tau(k) \text { for all } i>j
$$

In the deep LPM limit, where $\tau(k) \gg L_{i j}$, the destructive interference pattern summarized by the formation factor depends on the form of the current correlation functions. It is amusing to note that that the negative sign leading to destructive interference in eq. (71) arises in QCD from the color algebra, eq. (69), in contrast to QED where the destructive pattern in eq.(2) arises from the opposite sign of contributing momentum space amplitudes. 


\subsection{Current Correlation Function}

In order to investigate the formal structure of the current correlation function, eq.(66), we evaluate

$$
\begin{aligned}
\left\langle\vec{\jmath}_{i} \cdot \vec{\jmath}_{j}\right\rangle & \propto\left\langle\left(\frac{\mathbf{k}_{\perp}}{k_{\perp}^{2}}+\frac{\mathbf{q}_{\perp i}-\mathbf{k}_{\perp}}{\left|\mathbf{q}_{\perp i}-\mathbf{k}_{\perp}\right|^{2}}\right) \cdot\left(\frac{\mathbf{k}_{\perp}}{k_{\perp}^{2}}+\frac{\mathbf{q}_{\perp j}-\mathbf{k}_{\perp}}{\left|\mathbf{q}_{\perp j}-\mathbf{k}_{\perp}\right|^{2}}\right)\right\rangle_{+} \\
& \approx\left(1+H\left(k_{\perp}^{2}\right)\right)^{2} / k_{\perp}^{2},
\end{aligned}
$$

where in terms of the transverse vector, $\mathbf{J} \equiv\left(\mathbf{q}_{\perp}-\mathbf{k}_{\perp}\right) /\left|\mathbf{q}_{\perp}-\mathbf{k}_{\perp}\right|^{2}$

$$
H\left(k_{\perp}^{2}\right)=\left\langle\mathbf{k}_{\perp} \cdot \mathbf{J}\right\rangle_{+} \equiv \mathbf{k}_{\perp} \cdot\left\langle\frac{\mathbf{q}_{\perp}-\mathbf{k}_{\perp}}{\left|\mathbf{q}_{\perp}-\mathbf{k}_{\perp}\right|^{2}}\right\rangle_{+} .
$$

The approximate independence of the current correlations on the indices, $i, j$, is only valid in a kinematic region $k_{\perp}>x p_{\perp i}$ recalling eqs.(42). In QED this restriction is severe because the leading term, $\epsilon_{\perp} \cdot \mathbf{k}_{\perp} / k_{\perp}^{2}$, from eq.(42) cancels, and the photon spectrum is peaked at $x \sim 1$. In QCD, on the other hand, the radiated energy fraction, $x d n / d x d^{2} k_{\perp}$, is approximately independent of $x$ from eq.(62). Therefore, unlike in QED the regime $x \ll 1$ is relevant in the case of QCD. We find below that the induced radiation is indeed limited to $x<\lambda \mu^{2} / E_{0} \ll 1$, and thus the above approximation is justified. This approximation cannot however be extended outside the soft eikonal limit. For moderate $x<1$ it clearly breaks down especially because $p_{\perp i}^{2}$ grows approximately linearly with $i$ due to multiple scattering. For the general case, the exact current element must be used and the correlation function must be computed from a solution of a transport equation, as first done by Migdal[1] for QED. We limit the discussion here to the soft eikonal regime.

The diagonal correlator, in the same limit is proportional to the invariant gluon distribution from a single collision:

$$
\begin{aligned}
\left\langle\left|\vec{j}_{i}\right|^{2}\right\rangle & \propto\left\langle\left|\frac{\mathbf{k}_{\perp}}{k_{\perp}^{2}}+\frac{\mathbf{q}_{\perp}-\mathbf{k}_{\perp}}{\left|\mathbf{q}_{\perp}-\mathbf{k}_{\perp}\right|^{2}}\right|^{2}\right\rangle_{+}=\left\langle\frac{q_{\perp}^{2}}{k_{\perp}^{2}\left|\mathbf{q}_{\perp}-\mathbf{k}_{\perp}\right|^{2}}\right\rangle_{+} \\
& =\left(1+2 H\left(k_{\perp}^{2}\right)+H_{2}\left(k_{\perp}^{2}\right)\right) / k_{\perp}^{2},
\end{aligned}
$$


which involves a second function

$$
H_{2}\left(k_{\perp}^{2}\right)=k_{\perp}^{2}\left\langle|\mathbf{J}|^{2}\right\rangle_{+}=\left\langle\frac{k_{\perp}^{2}}{\left(\mathbf{q}_{\perp}-\mathbf{k}_{\perp}\right)^{2}}\right\rangle_{+} .
$$

The approximate correlation function in the soft eikonal limit is therefore independent of $i, j$ and given by

$$
F_{i j}(k) \approx F\left(k_{\perp}\right)=\frac{1+2 H+H^{2}}{1+2 H+H_{2}} .
$$

Because $\left\langle\left|\mathbf{J}-\left\langle\mathbf{k}_{\perp} \cdot \mathbf{J}\right\rangle / k_{\perp}^{2}\right|^{2}\right\rangle \geq 0$, note that $H_{2} \geq H^{2}$ and consequently the current correlation function is bounded:

$$
0 \leq F(k) \leq 1
$$

The upper bound is approached for $k_{\perp}^{2} \ll\left\langle q_{\perp}^{2}\right\rangle \sim \mu^{2}$. In that soft region both $H \approx H_{2} \propto k_{\perp}^{2} /\left\langle q_{\perp}^{2}\right\rangle \ll 1$, and

$$
F_{i j}(k) \approx F(0)=1
$$

The lower bound is approached in the opposite limit, $k_{\perp}^{2} \gg\left\langle q_{\perp}^{2}\right\rangle$, Formally, $H \approx$ $-1-\left\langle q_{\perp}^{2}\right\rangle / k_{\perp}^{2}$ while $H_{2} \approx 1+3\left\langle q_{\perp}^{2}\right\rangle / k_{\perp}^{2}$, and consequently in that limit

$$
F(k) \propto\left\langle q_{\perp}^{2}\right\rangle / k_{\perp}^{2}
$$

The exact form interpolating between these limits depends of course on the proper inclusion of polarization effects in the medium.

\subsection{The Factorization Limit}

For fixed $x \approx \omega / E_{0} \ll 1$ and $k_{\perp} \rightarrow 0, F_{i j} \approx 1$, and the formation length, $\tau(k)=$ $x P^{+} / k_{\perp}^{2}$ becomes much longer than the separation of the scattering centers. In this case, the phase factors can be set to unity. With the help of

$$
\sum_{i=1}^{m} \sum_{j=1}^{i-1}\left(1-r_{2}\right)^{i-j}=\frac{1-r_{2}}{r_{2}}\left(m-\frac{1}{r_{2}}\left(1-\left(1-r_{2}\right)^{m}\right)\right)
$$


the radiation formation factor reduces to

$$
\begin{aligned}
\lim _{k_{\perp} \rightarrow 0} C_{m}(k) & =C_{m}^{0}=\frac{1}{r_{2}}\left(1-\left(1-r_{2}\right)^{m}\right) \\
& =\left(\begin{array}{ll}
1-1 / N^{2}\left(1-1 /\left(1-N^{2}\right)^{m-1}\right) & \text { for quarks } \\
2\left(1-1 / 2^{m}\right) & \text { for gluons }
\end{array}\right.
\end{aligned}
$$

Note also that for a given $r_{2}=C_{A} / 2 C_{2}, C_{m}^{0}$ approaches $1 / r_{2}$, independent of the number of collisions as $m \rightarrow \infty$. This is the Factorization limit, in contrast to the additive Bethe-Heitler limit. The saturation value depends on the $S U(N)$ representation of the jet parton and causes the $C_{A}$ factor in eq. (62) to be replaced by $2 C_{2}$. Interestingly, for quarks in the fundamental representation the destructive interference is so effective that for $N=3$, the final radiation intensity in the $k_{\perp} \ll\left\langle q_{\perp}\right\rangle$ region after many collisions is even slightly less, $1 / r_{2}=8 / 9$, than for a single isolated collision. However, for incident gluon jets, the induced radiation approaches twice that from a single collision. For exotic hybrid partons in very high dimensional representations of $S U(N)$, the suppression effect in fact disappears altogether for fixed $m$ as $C_{A} / m C_{2} \rightarrow 0$. This dependence of the LPM effect on the representation of the parton is a specific non-abelian effect in QCD.

\subsection{Ensemble Averaged Formation Factor}

For $0<k_{\perp} \ll \mu$, we can write

$$
C_{m}\left(\omega, \mathbf{k}_{\perp}\right)=C_{m}^{0}+\frac{r_{2}}{1-r_{2}} R e \sum_{i=1}^{m} \sum_{j=1}^{i-1}\left(1-r_{2}\right)^{i-j}\left(1-F_{i j}(k) e^{i\left(z_{i}-z_{j}\right) / \tau(k)}\right)
$$

To see analytically how $C_{m}$ interpolates between $C_{m}^{0}$ and $m$ as a function of the ratio of the mean free path to the formation time, we average now over the interaction $z_{i}$ points according to linear kinetic theory. Because we restrict the discussion here to the eikonal case, the complication due to the full 3D transport evolution can be neglected. In linear kinetic theory the longitudinal separation between successive 
scatterings, $L_{i}=z_{i+1}-z_{i}$, is distributed simply as

$$
\exp \left(-L_{i} / \lambda\right) / \lambda
$$

where $\lambda=\left(\sigma_{0} \rho v_{0}\right)^{-1}$ is the mean free path. Therefore,

$$
\left\langle e^{i\left(z_{i}-z_{j}\right) / \tau(k)}\right\rangle \approx\left(\frac{1}{1-i \lambda / \tau(k)}\right)^{i-j}
$$

where $\tau(k)=2 x E_{0} / k_{\perp}^{2}$.

The sum in eq.(71) can then be performed since in this soft eikonal limit $F_{i j}(k) \approx$ $F(k) \approx 1$ In that case eq. 82 ) can be used with the replacement

$$
1-r_{2} \rightarrow \frac{1-r_{2}}{1-i \lambda / \tau(k)}
$$

The resulting radiation formation factor reduces to

$$
C_{m}(k)=m-\frac{m F(k)}{1+\chi^{2}(k)}+\frac{F(k)}{r_{2}} \operatorname{Re}\left(\frac{\left(1-i r_{2} \chi(k)\right)}{(1-i \chi(k))^{2}}\left[1-\left(\frac{1-r_{2}}{1-i r_{2} \chi(k)}\right)^{m}\right]\right)
$$

where the dimensionless function controlling the non-abelian LPM effect is

$$
\chi(k)=\frac{\lambda}{\tau(k) r_{2}}=\frac{\lambda k_{\perp}^{2}}{2 x r_{2} E_{0}}=\frac{C_{2}}{C_{A}} \frac{\lambda k_{\perp}}{\cosh (y)} .
$$

The last form is in terms of the rapidity, $y$ of the radiated gluon $\left(\omega=k_{\perp} \cosh y\right)$. Note that eq.(88) satisfies all the previous limits considered $\left(m=1, k_{\perp}=0, \lambda=\infty\right)$.

For moderate large $m$ the term proportional to $\left(1-r_{2}\right)^{m}$ term can be neglected, and the formation factor simplifies to

$$
C_{m}\left(x, k_{\perp}\right) \approx m\left(1-\frac{F(k)}{1+\chi^{2}(k)}\right)+\frac{F(k)}{r_{2}}\left(\frac{1-\left(1-2 r_{2}\right) \chi^{2}(k)}{\left(1+\chi^{2}(k)\right)^{2}}\right) .
$$

This illustrates clearly how the radiation formation factor interpolates between the $\tau=0$ and $\tau=\infty$ limits as a function of the dimensionless variable $\chi$. However, it also shows that, through the dependence on the current correlation function, $F(k)$, the radiation formation factor is actually a function of two dimensionless variables, $\chi(k)=\lambda / r_{2} \tau$ and $k_{\perp}^{2} / \mu^{2}$. Thus, both the range, $\mu^{-1}$, as well as the separation, $\lambda$, of the interactions influences the final interference pattern. 
For quarks, $r_{2} \approx 1$, while for gluons $r_{2}=1 / 2$. In the collinear regime, $k_{\perp} \ll \mu$ regime, $F(k) \approx 1$, and a further simplification occurs. The radiation formation factors for incident quarks and gluons reduce then to the following simple "pocket" formulas

$$
\begin{aligned}
C_{m}^{q}(k) & \approx \frac{\tau^{2}(k)+m \lambda^{2}}{\tau^{2}(k)+\lambda^{2}} ; \\
C_{m}^{g}(k) & \approx \frac{4 m \lambda^{2}}{\tau^{2}(k)+4 \lambda^{2}}+\frac{2}{\left(\tau^{2}(k)+4 \lambda^{2}\right)^{2}} .
\end{aligned}
$$

While the above interpolation formula for quarks can be extrapolated down to $m=1$, the gluon one only applies for large $m$ because of the extra factor of two radiation in that case for large $m$. We emphasize again the restriction $x \mu \sqrt{ } m \ll k_{\perp} \ll \mu$ used in deriving the above expressions.

\section{$5 \quad$ Induced Soft Radiative Energy Loss}

An important application of the radiation formation factor is to the problem of calculation of the radiative energy loss per unit length, $d E / d z$, for a parton passing through dense, color neutral matter with a mean free path, $\lambda \gg \mu^{-1}$. We need only the incremental increase of the induced radiation going from $m$ to $m+1$. For moderately large $m$ and $k_{\perp}<\mu$

$$
d C_{m} / d m \approx \chi^{2}(k) /\left(1+\chi^{2}(k)\right)
$$

with $\chi(k)$ given by eq.(89). Increasing $m \rightarrow m+1$, the average increase of the interaction length is $\lambda$, and thus from eq. (62, 63)

$$
d E_{\text {soft }} / d z=\frac{d}{\lambda d m} \int d n_{g} \omega \approx \int_{0}^{\mu} d k_{\perp}^{2} \int_{x_{0}}^{x_{1}} \frac{d x}{x} \frac{\alpha_{s} C_{A}}{\pi \lambda k_{\perp}^{2}} \frac{x E_{0} \chi^{2}\left(x, k_{\perp}\right)}{1+\chi^{2}\left(x, k_{\perp}\right)}
$$

The subscript "soft" is included to emphasize that the eikonal approximation, used in deriving eq.(92), restricts its applicability to the kinematic region $x \mu \sqrt{ } m<k_{\perp}<$ $\mu$. The limits on the fractional energy loss are $x_{0} \approx k_{\perp} / E_{0}$ from kinematics, and

$x_{1} \sim k_{\perp} / \mu$ from the above restriction. Note that we neglect the $1 / \sqrt{ } m$ dependence 
of $x_{1}$ since the Factorization limit is found to be insensitive to this cutoff, while the Bethe-Heitler limit must reduce in any case just the sum the radiation from isolated $(m=1)$ collisions.

The kinematic restriction $k_{\perp}^{2}<\mu^{2}$, also follows from the destructive interference between three gluon vertex and jet line amplitudes. This point was already emphasized in [19] but was missed in ref.[13], where the domain of $k_{\perp}$ integration was allowed to extend up to the kinematic limit, $k_{\perp}^{2} \leq s / 4=E_{0} m / 2$. That lead to the erroneous conclusion that $d E / d z \propto E_{0}$, in violation with the factorization theorems.

The integral over $x$ can be performed by changing variables to $\chi$, with the result

$$
\int_{x_{0}}^{x_{1}} \frac{d x}{x} \frac{x E_{0} \chi^{2}}{1+\chi^{2}}=\lambda_{2} k_{\perp}^{2}\left(\tan ^{-1}\left(\lambda_{2} k_{\perp}\right)-\tan ^{-1}\left(\lambda_{2} k_{\perp}\left(\mu / E_{0}\right)\right)\right)
$$

where $\lambda_{2} \equiv \lambda / 2 r_{2}$. We see that in the $E_{0} \gg \lambda_{2} \mu^{2}$ limit, the second term is negligible. Furthermore, since $\lambda \mu \sim 1 / g \gg 1$ is a basic assumption in our multiple collision analysis, the first term in the brackets is approximately $\pi / 2$.

The integral over $k_{\perp}$ is also analytic and illustrates how $d E / d z$ interpolates between the Factorization and Bethe-Heitler limits. We find that

$$
d E_{\text {soft }} / d x \approx \frac{1}{2} \alpha_{s} C_{2} \mu^{2}\left\{L\left[\lambda_{2} \mu\right]-L\left[\lambda_{2} \mu\left(\mu / E_{0}\right)\right]\right\}
$$

where the interpolation function is

$$
L[a]=\frac{2}{\pi}\left[\left(1+\frac{1}{a^{2}}\right) \tan ^{-1}(a)-\frac{1}{a}\right] .
$$

Note that for $a \gg 1, L[a] \approx 1-4 /(\pi a)$. For $a \ll 1$, on the other hand, $L[a] \approx 4 x / 3 \pi$. Since, $\lambda_{2} \mu \gg 1$, the first term in the brackets is always close to unity. However, the second term depends on the dimensionless ratio, $\zeta \equiv \lambda \mu^{2} / E_{0}$. This ratio is large in the additive Bethe-Heitler limit and small in the Factorization limit.

To see how eq.(95) interpolates between those two limits consider first the approximate Factorization limit. We fix $\lambda_{2} \mu \gg 1$ and send $E_{0} \rightarrow \infty$. In that case, the second term in eq.(95) can be neglected and

$$
d E_{\text {soft }} / d z \approx \frac{1}{2} \alpha_{s} C_{2} \mu^{2} \approx 2 \pi \alpha_{s}^{2} C_{2} T^{2},
$$


where we used $\mu \sim g T$ to estimate the force range in a quark-gluon plasma at temperature $T$. The result is thus sensitive to the square of the radiated transverse momentum as suggested in 99. Note that because we are only calculating the low $k_{\perp}<\mu$ contribution, our derivation does not allow us to calculate logarithmic energy dependent factors as obtained qualitatively in [9]. However, up to such logarithmic factors eq.(97) demonstrates the approximate constant behavior of the induced radiated energy loss in the Factorization limit.

In the other extreme limit, we fix $E_{0}$ and send $\lambda \mu \rightarrow \infty$ so that $\zeta \gg 1$. This is the dilute limit where the mean free path exceeds the radiation formation length. In this case the arguments of both terms in eq.95) are large, and the small difference leads to

$$
d E_{\text {soft }} / d z \sim \frac{1}{2} \alpha_{s} C_{2} \mu^{2} \frac{4 E_{0}}{\pi \mu^{2} \lambda_{2}} \sim \frac{E_{0}}{\lambda}\left(\frac{2 \alpha_{s} C_{A}}{\pi}\right)
$$

Note that in this limit we recover the linear dependence of $d E / d z$ on the incident energy (modulo logarithms), as in the Bethe-Heitler formula.

It is interesting to note that in the additive regime the radiated energy loss is proportional to $C_{A}$, as for a single scattering via eq.(62), However, in the approximate Factorization limit the induced radiated energy loss is proportional to the $C_{2}$ of the jet parton. This means in practice that gluons radiate $C_{A} / C_{F}=9 / 4$ more gluons than quarks for $S U(3)$. Recall, that the energy loss due to elastic collisions for gluons is also enhanced relative to quarks by the same $C_{A} / C_{F}$ factor[21]. For comparison, the energy loss due to elastic collisions from [21] is

$$
d E_{e l} / d z \approx \frac{4 \pi}{3} C_{2} \alpha_{s}^{2} T^{2} \log \left(E_{0} / \pi \alpha_{s} T\right)
$$

Therefore, the total $d E / d x$ simply scales with $C_{2}$. This scaling differs from the qualitative estimates in [9] using the single scattering bremsstrahlung cross sections of [19]. It is interesting to note that both the elastic and radiated energy loss is proportional to $\alpha_{s}^{2}$ and are comparable in magnitude up to uncertain logrithmic factors.

We emphasize that eq.(97) for the radiated energy loss is only an order of magnitude estimate because we have not calculated the contribution from the non-factoring 
$k_{\perp}>\mu$ domain. In order to improve the estimate, all the multiple three gluon vertex amplitudes added to those computed here. That moderate high $k_{\perp}$ regime also requires a more careful treatment of the current correlation function as well as of the polarization mechanisms that regulate infrared singularities. A proper treatment of the above problems remains an open theoretical challenge.

Finally, we comment on the comparison of eq.(97) to the bound on $d E / d x$ derived by Brodsky and Hoyer [10] based on the uncertainty principle. For a radiated gluon carrying away a fraction $x$ of the incident energy, $E_{0}$, with a given $k_{\perp}$, the uncertainty in its formation length is $\tau(k)$ from eq.(1). The induced energy loss for radiating one gluon is therefore bounded by

$$
d E / d x<\langle x E / \tau(k)\rangle=\left\langle k_{\perp}^{2}\right\rangle / 2 \sim \mu^{2} / 2
$$

Our estimate satisfies this bound because $\alpha_{s} \ll 1$ was assumed throughout our perturbative analysis. In fact, we may interpret $C_{2} \alpha_{s}$ roughly the probability of radiating one gluon with $\tau(k)<\lambda$ between multiple collisions. That gluon is radiated in a cylindrical phase space with approximate uniform rapidity density between $0<y<\log \left(\mu \lambda / r_{2}\right)$ and limited $k_{\perp}<\mu$.

\section{Summary and Discussion}

In this paper, we initiated a study of multiple collision theory in pQCD concentrating on the eikonal limit. We calculated elastic and inelastic multiple collision amplitudes for a high energy parton propagating though a "plasma" of static target partons. We showed that the assumption of color neutrality was vital to recover the classical parton cascade picture in both large and small angle scattering. Our main simplifying assumption was that the mean free path $\left(\lambda \sim 1 / g^{2} T\right)$ in the target was large compared to the range of the interactions $\left(\mu^{-1} \sim 1 / g T\right)$. In particular, we showed how the classical Glauber scattering cross section eq.(33) emerges after ensemble averaging.

The main focus of the paper was to derive eq.(57), which shows how the sum of the induced gluon radiation amplitudes can be expressed as a convolution of elastic multiple scattering amplitudes and an effective color current, eq.(58). This result was 
derived for the soft radiation $\left(x \ll 1\right.$ and $\left.k_{\perp} \ll \mu\right)$ regime. The limitations of the approximations leading to that result were also carefully analyzed. We showed that the triple gluon diagrams can be neglected in the soft limit but are important to cut off the $k_{\perp}$ distributions on the scale $\mu$. Only when $k_{\perp} \rightarrow 0$ can the effective current be pulled out of the multiple collision integral, and the radiation amplitude factored in momentum space as in eq.(49). There is never a factorization of amplitudes in color space. However, the color neutrality condition, eq.23), greatly simplifies the ensemble average of the squared amplitude. In addition, the off diagonal contributions in momentum space from $\overrightarrow{J_{a_{1} \cdots a_{m}}^{c}}\left(k ;\left\{\mathbf{q}_{\perp i}\right\}\right) \vec{J}_{a_{1} \cdots a_{m}}^{c}\left(k ;\left\{\mathbf{q}_{\perp i}^{\prime}\right\}\right)$ drop out, as shown below eq.(31), if the transverse width of the target is large compared to the interaction range. Under these conditions, it was possible to calculate the induced radiation spectrum from eq. 60 .

We defined the radiation formation factor, eq.(64), as the ratio of the induced radiation spectrum to the spectrum from an isolated collision. That factor measures the suppression of induced radiation with formation length, $\tau(k)>\lambda$, and reveals the non-abelian analog of the LPM effect. The novel role of the color algebra that leads to this destructive interference pattern in QCD was shown in Eqs. (69.71). We showed how this factor interpolates between the saturated Factorization limit and the additive Bethe-Heitler limit. A compact "pocket" formula for the formation factor was derived in eq.(91) illustrating the essential features of that interpolation.

Finally, we applied the formation factor to estimate the contribution of soft induced gluon radiation to the energy loss per unit length, eq.(95). The result in the Factorization limit, eq.(97), was shown to be consistent with the uncertainty principle bound of [10] with a numerical coefficient, $C_{2} \alpha_{s}$, that had a simple physical interpretation as the number of induced gluons radiated in the limited phase space with rapidity between zero and $\log \left(\mu \lambda / r_{2}\right)$ and with $k_{\perp}<\mu$. Up to un-calculated logarithmic factors the radiative energy loss was found to be comparable to the elastic energy loss 21]. We also showed how the linear energy dependence of $d E / d z$ is recovered in the opposite (Bethe-Heitler) limit when $\lambda \gg E / \mu^{2}$.

Naturally, many problems need further study. Especially important will be to ex- 
tend the derivation of radiative cross sections to the moderate $k_{\perp}>\mu$ regime to cover the non-factorizable "semi-hard" regime for induced radiation. We concentrated on the soft regime here to simplify our task. The "semi-hard" regime is however also complicated by the necessity of having to consider in detail the polarization effects that regulate $\mathbf{q}_{\perp}=\mathbf{k}_{\perp}$ singularities and also the necessity of computing the current correlation functions discussed in section 4.2. The open theoretical question in this connection is to what extent, if any, can a classical parton cascade transport model be constructed that correctly simulates the many subtle interference phenomena of pQCD in the multiple collision domain.

Acknowledgements: Stimulating discussions with S. Brodsky, A. Mueller, B. Muller, M. Grabiak, Sovand P. Danielewicz are gratefully acknowledged. This work was initiated while M.G. was at LBL and X.N. Wang was at Duke University.

\section{References}

[1] L.D. Landau and I.J. Pomeronchuk, Dolk.Akad.Nauk. SSSR 92 (1953) 92; A.B. Migdal, Phys. Rev. 103 (1956) 1811; E. L. Feinberg and I.J. Pomeronchuk, Suppl. Nuovo. Cimento 3 (1956) 652; . Phys. JETP 23 (1966) 132; J.S. Bell, Nucl. Phys. 8 (1958) 613; N.N. Nikolaev, Sov. Phys. Ups. 24 (1981) 531.

[2] A.I. Akhiezer and N.F. Shulga, Sov. Phys. Usp. 30 (1987) 197.

[3] Perturbative Quantum Chromodynamics, ed. A.H. Mueller, (Advanced Series on Directions of High Energy Physics, Vol 5, World Scientific, 1989).

[4] A. Bassetto, M. Ciafaloni, and G. Marchesini, Phys. Rep. 100 (1983) 201.

[5] G. T. Bodwin, S. J. Brodsky, and G. P. Lepage, Phys. Rev. Lett. 47 (1981) 1799.

[6] D.M. Alde et al., Phys. Rev. Lett. 64 (1990) 2479 ; 66 (1991) 133; 2285. 
[7] European Muon Collaboration (J. Ashman, et al.) Z.Phys.C52:1-12,1991; N.A. Pavel Nucl. Phys. A532 (1991) 465c-488c; A. F. Salvarani (E665 Colab.), Ph.D.Thesis UCSD, UMI-91-30738-mc (microfiche), 1991. 318pp.

[8] M. Gyulassy and M. Plümer, Nucl. Phys. B346 (1990) 1. D. J. Dean, et al., Phys. Rev. C46 (1992) 2066.

[9] M. Gyulassy, et al. Nucl. Phys. A 538 (1992) 37c.

[10] S.J. Brodsky and P. Hoyer, Phys. Lett. B298 (1993) 165.

[11] A. H. Sorensen, Z. Phys. C53 (1992) 595.

[12] S. Gavin, J. Milana Phys.Rev.Lett.68:1834-1837,1992.

[13] M. G. Ryskin, Sov. J. Nucl. Phys., 52 (1990) 139.

[14] B. Andersson, et. al., Nucl. Phys. B281 (1987) 289; J. Ranft, Phys. Lett. B188 (1987) 379; K. Werner, et .,Phys. Lett. 183B (1987) 385; R. Mattiello et al, Phys. Rev. Lett. 63 (1989) 1459; H. Sorge et al, Nucl. Phys. A525 (1991) 95c.

[15] X.N. Wang and M. Gyulassy, Phys.Rev. D44 (1991) 3501; D45 (1992) 844; Phys. Rev. Lett. 68 (1992) 1480.

[16] H.T. Elze and U. Heinz, Phys. Rep.183 (1989) 81; S. Mrowczynski; M. Gyulassy, in Quark-Gluon Plasma, Advanced Series on Directions In High Energy Physics, Vol. 6, ed. R. C. Hwa (World Scientific,1990) pp.185, 223; A.V. Selikhov, Phys. Lett. B268 (1991) 263.

[17] T. Stanev, et al., Phys. Rev. D25 (1985) 1291.

[18] M. Cavalli-Sforza et al., SLAC-Proposal-E146 (1992).

[19] J.F. Gunion and G. Bertsch, Phys. Rev. D25, 746 (1982).

[20] H.A. Weldon, Phys. Rev.D26 (1982) 1394; A. V. Selikhov, LBL-32783 (1992) preprint, unpublished.

[21] M. H. Thoma, M. Gyulassy, Nucl. Phys. B351 (1991)491. 


\section{Appendix A: Large Angle Elastic Cascade Limit}

We consider here the elastic coincidence scattering in the special idealized case that the coordinates $\mathbf{x}_{i}$ are fixed and the energy is large to see how the classical billiard ball formula emerges. We rewrite the phases in eq.(19) as

$$
\mathbf{p}_{k} \cdot \mathbf{R}_{k} \approx \mathbf{P}_{k} \cdot \mathbf{R}_{k}-\frac{L_{k}}{2 P_{0}}\left(\mathbf{p}_{\perp k}-\mathbf{P}_{\perp k}\right)^{2}
$$

with $\mathbf{P}_{k}=P_{0} \mathbf{R}_{k} / R_{k}$ and

$$
\mathbf{P}_{\perp k}=P_{0} \mathbf{r}_{\perp k} / R_{k} \approx P_{0} \mathbf{r}_{\perp k} / L_{k}
$$

Note that $\mathbf{p}_{\perp k} \cdot \mathbf{r}_{\perp k}=2 \mathbf{p}_{\perp k} \cdot \mathbf{P}_{\perp k}\left(L_{k} / 2 P_{0}\right)$ and $P_{0} L_{k} \approx \mathbf{P}_{k} \cdot \mathbf{R}_{k}-\mathbf{P}_{\perp k}^{2}\left(L_{k} / 2 P_{0}\right)$.

Substituting eq.(101) into eq.(19) and shifting the $\mathbf{p}_{\perp k}$ integration, we find that

$$
\begin{aligned}
I_{j i}\left(p_{j}, p_{i-1}\right) \approx e^{+i \phi_{j i}} \int & \left\{\prod_{k=i}^{j-1} \frac{d^{2} \mathbf{p}_{\perp k}}{(2 \pi)^{2}} \frac{e^{-i \frac{L_{k}}{2 P_{k}} \mathbf{p}_{\perp k}^{2}}}{2 P_{0}}\right\} A_{j}\left(\mathbf{Q}_{j}-\mathbf{p}_{\perp j-1}\right) \\
& \times\left\{\prod_{l=i}^{j-1} A_{l}\left(\mathbf{Q}_{l}+\mathbf{p}_{\perp l}-\mathbf{p}_{\perp(l-1)}\right)\right\}
\end{aligned}
$$

where the intermediate classical momentum transfers, denoted by

$$
\mathbf{Q}_{k}=\mathbf{P}_{k}-\mathbf{P}_{k-1} \text { for } i<k<j
$$

have dominantly transverse components. The endpoint momentum transfers are given $\mathbf{Q}_{j}=\mathbf{p}_{j}-\mathbf{P}_{j-1}$ and $\mathbf{Q}_{i}=\mathbf{P}_{i}-\mathbf{p}_{i-1}$, and the external phase is

$$
\phi_{j i}=\sum_{l=i}^{j-1} \mathbf{P}_{l} \cdot \mathbf{R}_{l}=\sum_{l=i}^{j} \mathbf{Q}_{l} \cdot \mathbf{x}_{l}+i \mathbf{p}_{j} \cdot \mathbf{x}_{j}-i \mathbf{p}_{i-1} \cdot \mathbf{x}_{i-1} .
$$

A simplification occurs in the high energy when the $\mathbf{x}_{i}$ are fixed because the $Q_{\perp k}$ increase linearly with $E_{0}$, while the transverse momentum integrals are limited by the oscillating phase factors to $p_{\perp k} \lesssim\left(P_{0} / L_{k}\right)^{1 / 2}$. Hence $Q_{\perp k} \gg p_{\perp k}$ for high enough 
energies, and we can expand the potentials around $\mathbf{Q}_{\perp k}$. This is equivalent to the stationary phase approximation. The region of validity of that approximation can be clarified by writing

$$
\mathbf{Q}_{\perp k}=P_{0}\left(\mathbf{r}_{\perp k} / L_{k}-\mathbf{r}_{\perp k-1} / L_{k-1}\right) \equiv P_{0} \Delta \mathbf{r}_{\perp k} / L_{k}
$$

The condition that $Q_{\perp k} \gg\left(P_{0} / L_{k}\right)^{1 / 2}$ is thus equivalent to requiring that the transverse momentum transfer be large enough to resolve the transverse separation of the scattering centers:

$$
\mathrm{Q}_{\perp k} \cdot \Delta \mathbf{r}_{\perp k} \gg \hbar
$$

For fixed $\mathbf{r}_{\perp k}$ this condition is always satisfied for sufficiently large energies, and thus the stationary phase integrals can then be evaluated using

$$
\int \frac{d^{2} \mathbf{p}_{\perp}}{2 \pi i P} e^{-i p_{\perp}^{2} L / 2 P}=\frac{1}{L}
$$

In this limit $M_{j i}$ reduces to the simple factorized form

$$
M_{j i}\left(p_{j}, p_{i-1}\right) \approx \delta(j i) e^{+i \mathbf{Q}_{j} \cdot \mathbf{x}_{j}} A_{j}\left(\mathbf{Q}_{j}\right) \prod_{k=i}^{j-1}\left\{e^{i \pi / 2} e^{+i \mathbf{Q}_{k} \cdot \mathbf{x}_{k}} A_{k}\left(\mathbf{Q}_{k}\right) /\left(4 \pi R_{k}\right)\right\}
$$

Note again that in the non-Abelian case the matrix ordering from $j$ to $i$ is essential.

After squaring and integrating over the magnitude of the final momentum, averaging over initial colors and summing over final, the above factorized form leads via eq.(11) to the classical billiard ball formula

$$
d \sigma_{j i} / d \Omega=d \sigma_{i} / d \Omega_{i}\left\{\prod_{k=i+1}^{j} d \sigma_{k} / R_{k}^{2} d \Omega_{k}\right\}
$$

We emphasize that color neutrality of the medium and large transverse momentum transfers are essential to recover this simple cascade limit in which the direction of all intermediate momenta are fixed by geometry. 


\section{Appendix B: Derivation of Eq.(39)}

Technical details of the derivation of the radiation amplitude from intermediate jet lines are given here. The integration over $p_{z j}$ in eq.(35) can be expressed as a sum of two terms using eq.(38). The term containing the $\Delta\left(p_{j}-k\right)$ propagator leads to

$$
\begin{aligned}
M_{c j}^{+}=i g \tilde{\delta}(m 1) e^{-i \mathbf{p}_{m} \cdot \mathbf{x}_{m}} e^{+i \mathbf{p}_{0} \cdot \mathbf{x}_{1}} \int \frac{d^{3} p_{j}}{(2 \pi)^{3}} \frac{e^{+i \mathbf{p}_{j} \cdot \mathbf{R}_{j}} e^{-i \mathbf{k} \cdot \mathbf{x}_{j+1}}}{P_{\omega}^{2}-\left(\mathbf{p}_{j}-\mathbf{k}\right)^{2}+i \epsilon} \\
\times \frac{\epsilon\left(p_{j}-k\right)}{k\left(p_{j}-k\right)} I_{m, j+1}\left(p_{m}, p_{j}-k\right) T_{c} I_{j, 1}\left(p_{j}, p_{0}\right) .
\end{aligned}
$$

The term containing the $\Delta\left(p_{j}\right)$ propagator leads to

$$
\begin{gathered}
M_{c j}^{-}=-i g \tilde{\delta}(m 1) e^{-i \mathbf{p}_{m} \cdot \mathbf{x}_{m}} e^{+i \mathbf{p}_{0} \cdot \mathbf{x}_{1}} \int \frac{d^{3} p_{j}}{(2 \pi)^{3}} \frac{e^{+i \mathbf{p}_{j} \cdot \mathbf{R}_{j}} e^{-i \mathbf{k} \cdot \mathbf{x}_{j+1}}}{P_{0}^{2}-\mathbf{p}_{j}^{2}+i \epsilon} \\
\times \frac{\epsilon p_{j}}{k p_{j}} I_{m, j+1}\left(p_{m}, p_{j}-k\right) T_{c} I_{j, 1}\left(p_{j}, p_{0}\right) .
\end{gathered}
$$

In $M_{c j}^{+}$the contour integral over $p_{z j}$ sets $p_{j}-k$ on shell with

$$
\begin{aligned}
p_{z j} & \approx k_{z}+P_{\omega}-\left(\mathbf{p}_{\perp j}-\mathbf{k}_{\perp}\right)^{2} / 2 P_{\omega} \\
& \approx P_{0}-\left(\mathbf{p}_{\perp j}-\mathbf{k}_{\perp}\right)^{2} / 2 P_{0}+\left(k_{z}-\omega / v_{0}\right)
\end{aligned}
$$

while in $M_{c j}^{-}$it sets $p_{j}$ on shell with $p_{z j} \approx P_{0}-\mathbf{p}_{\perp j}^{2} / 2 P_{0}$. In both cases the residue of the propagator can be well approximated by $1 / 2 P_{0}$ in the high energy limit when $x \ll 1$. However, it is essential to keep track of the difference, $P_{0}-P_{\omega}=\omega / v_{0}$ in computing the phases. The phase in the integrand of $M_{c j}^{+}$is given by

$$
\begin{aligned}
\mathbf{p}_{j} \cdot \mathbf{R}_{j}-\mathbf{k} \cdot \mathbf{x}_{j+1} & =\left(\mathbf{p}_{j}-\mathbf{k}\right) \mathbf{R}_{j}-\mathbf{k} \cdot \mathbf{x}_{j} \\
& \approx \mathbf{P}_{j} \cdot \mathbf{R}_{j}-\frac{L_{j}}{2 P_{0}}\left(\mathbf{p}_{\perp j}-\mathbf{k}_{\perp}-\mathbf{P}_{\perp j}\right)^{2}-\omega R_{j} / v_{0}-\mathbf{k} \cdot \mathbf{x}_{j}
\end{aligned}
$$

Note that the replacement $\omega L_{j} / v_{j} \rightarrow \omega R_{j} / v_{j}$ above is valid either when $r_{\perp j} \sim d \ll L_{j}$ or $P_{0} \rightarrow \infty$. The phase in $M_{c j}^{-}$is, on the other hand,

$$
\mathbf{p}_{j} \cdot \mathbf{R}_{j}-\mathbf{k} \cdot \mathbf{x}_{j+1} \approx \mathbf{P}_{j} \cdot \mathbf{R}_{j}-\frac{L_{j}}{2 P_{0}}\left(\mathbf{p}_{\perp j}-\mathbf{P}_{\perp j}\right)^{2}-\mathbf{k} \cdot \mathbf{x}_{j+1}
$$


Shifting the $\mathbf{p}_{\perp j}$ integration to $\mathbf{p}_{\perp j}+\mathbf{k}_{\perp}+\mathbf{P}_{\perp j}$ and $\mathbf{p}_{\perp j}+\mathbf{P}_{\perp j}$ in $M_{c j}^{ \pm}$respectively,

$$
\begin{aligned}
M_{c j}^{+} \approx g \tilde{\delta} & (m 1) e^{-i \mathbf{p}_{m} \cdot \mathbf{x}_{m}} e^{+i \mathbf{p}_{0} \cdot \mathbf{x}_{1}} \int \frac{d^{2} \mathbf{p}_{\perp j}}{(2 \pi)^{2}} \frac{1}{2 P_{0}} e^{i \mathbf{P}_{j} \cdot \mathbf{R}_{j}} e^{-i \frac{L_{j}}{2 P_{0}} \mathbf{p}_{\perp j}^{2}} \\
& \times\left(\frac{\epsilon \tilde{p}_{j}}{k \tilde{p}_{j}} e^{-i\left(\omega R_{j} / v_{j}+\mathbf{k} \cdot \mathbf{x}_{j}\right)}\right) I_{m, j+1}\left(p_{m}, \tilde{p}_{j}\right) T_{c} I_{j, 1}\left(p_{j}, p_{0}\right) \\
M_{c j}^{-} \approx- & -g \tilde{\delta}(m 1) e^{-i \mathbf{p}_{m} \cdot \mathbf{x}_{m}} e^{+i \mathbf{p}_{0} \cdot \mathbf{x}_{1}} \int \frac{d^{2} \mathbf{p}_{\perp j}}{(2 \pi)^{2}} \frac{1}{2 P_{0}} e^{i \mathbf{P}_{j} \cdot \mathbf{R}_{j}} e^{-i \frac{L_{j}}{2 P_{0}} \mathbf{p}_{\perp j}^{2}} \\
& \times\left(\frac{\epsilon p_{j}}{k p_{j}} e^{-i \mathbf{k} \cdot \mathbf{x}_{j+1}}\right) I_{m, j+1}\left(p_{m}, p_{j}-k\right) T_{c} I_{j, 1}\left(p_{j}, p_{0}\right) .
\end{aligned}
$$

Note that in $M_{c j}^{-}$, the on-shell $p_{j}$ and $\tilde{p}_{j}$ are given by Eqs.40,41). Therefore both the off-shell amplitude $I_{m, j+1}\left(p_{m}, p_{j}-k\right)$ and the on-shell amplitude $I_{m, j+1}\left(p_{m}, \tilde{p}_{j}\right)$ in the integrands above are evaluated with the same shifted incident energy, $E_{0}-\omega$, and approximately the same shifted incident longitudinal momentum, $P_{\omega} \approx P_{0}-\omega / v_{0}$.

The energy shift of $I_{m, j+1}$ above leads in the high energy limit to a phase shift of those amplitudes relative to the case without radiation. To see this, note from eq.(17) that

$$
I_{m, j+1}\left(p_{m}, p_{j}-k\right)=\int\left\{\prod_{k=j+1}^{m-1} \frac{d^{3} \mathbf{p}_{k}}{(2 \pi)^{3}} \frac{e^{i \pi / 2} e^{+i \mathbf{p}_{k} \cdot \mathbf{R}_{k}}}{P_{\omega}^{2}-\mathbf{p}_{k}^{2}+i \epsilon}\right\} A_{m}\left(\mathbf{p}_{m}-\mathbf{p}_{m-1}\right) \cdots A_{j+1}\left(\mathbf{p}_{j+1}-\mathbf{p}_{j}+\mathbf{k}\right)
$$

The contour integrals over the $p_{z k}$ then fix the phases to be

$$
\begin{aligned}
\mathbf{p}_{k} \cdot \mathbf{R}_{k} & \approx P_{\omega} L_{k}-p_{\perp k}^{2}\left(L_{k} / 2 P_{\omega}\right)+\mathbf{p}_{\perp k} \cdot \mathbf{r}_{\perp k} \\
& \approx \mathbf{P}_{k} \cdot \mathbf{R}_{k}-\frac{L_{k}}{2 P_{0}}\left(\mathbf{p}_{\perp k}-\mathbf{P}_{\perp k}\right)^{2}-\omega R_{k} / v_{0}
\end{aligned}
$$

Therefore, there is an additional phase shift $\omega L_{k} / v_{0} \approx \omega R_{k} / v_{0}$ for each intermediate line. This phase shift has a simple physical interpretation. Noting that the classical transit time between centers at $\mathbf{x}_{k}$ and $\mathbf{x}_{k+1}$ is

$$
\Delta t_{k} \approx R_{k} / v_{0}
$$


the additional phase shift is due to the time delay of a wavefront propagating with a frequency reduced by $\omega$. Defining the classical interaction time at center, $k$, as

$$
t_{k}=\sum_{i=1}^{k-1} \Delta t_{i}
$$

with $t_{1} \equiv 0$, the net extra phase can be factored out from eq.(118) as

$$
\begin{array}{r}
I_{m, j+1}\left(p_{m}, p_{j}-k\right) \approx e^{-i \omega\left(t_{m}-t_{j+1}\right)} e^{+i \phi_{m, j+1}} \int\left\{\prod_{k=j+1}^{m-1} \frac{d^{2} \mathbf{p}_{\perp k}}{(2 \pi)^{2}} \frac{e^{-i \frac{L_{k}}{2 P_{0}}\left(\mathbf{p}_{\perp k}-\mathbf{P}_{\perp k}\right)^{2}}}{2 P_{0}}\right\} \\
\times A_{m}\left(\mathbf{p}_{m}-\mathbf{p}_{m-1}\right) \cdots A_{j+1}\left(\mathbf{p}_{j+1}-\mathbf{p}_{j}+\mathbf{k}\right),
\end{array}
$$

where $\phi_{m, j+1}$ is the phase without radiation given via eq.(105). In eq.(122) we again used the condition $x \ll 1$ in replacing the residue $1 / P_{\omega}$ by $1 / P_{0}$.

Note that the longitudinal momentum transfer in all the potential is still small since all the intermediate longitudinal momenta are shifted by approximately $k_{z}$. If in addition to $x \ll 1$, we consider radiation with $k_{\perp} \ll \mu$, then the arguments of all the potentials inside the integrand can be approximated by those in eq.(19). Only the extra $\omega$ dependent phase must be kept. Therefore, in this soft limit

$$
I_{m, j+1}\left(p_{m}, p_{j}-k\right) \approx e^{-i \omega\left(t_{m}-t_{j+1}\right)} I_{m, j+1}\left(p_{m}, p_{j}\right)
$$

where the right hand side is to be evaluated ignoring the soft radiation via eq.(19). Similarly, it follows that for soft radiation

$$
I_{m, j+1}\left(p_{m}, \tilde{p}_{j}\right) \approx e^{-i \omega\left(t_{m}-t_{j+1}\right)} I_{m, j+1}\left(p_{m}, p_{j}\right)
$$

involving the same phase shift as in eq.(123). Combining these results we obtain eq.(39).

\section{Appendix C: Derivation of Eq.(56)}

The details of the derivation of the radiation amplitudes involving one three gluon vertex are given here. From eq. 26)

$$
M_{j-1,1}\left(p_{j-1}, p_{0}\right)=2 \pi \delta\left(p_{j-1}^{0}-E^{0}\right)\left(a_{j-1} \cdots a_{1}\right)(-i g)^{j-1}\left(2 E_{0}\right) e^{-i\left(p_{z(j-1)}-P_{0}\right) z_{j-1}}
$$




$$
\begin{gathered}
\times \int \prod_{k=1}^{j-1}\left(\frac{d^{2} q_{\perp k}}{(2 \pi)^{2}} e^{-i \mathbf{q}_{\perp k} \cdot \mathbf{x}_{\perp k}} V_{k}^{a_{k}}\left(\mathbf{q}_{\perp k}\right)\right)(2 \pi)^{2} \delta\left(\mathbf{p}_{\perp(j-1)}-\sum_{k=1}^{j-1} \mathbf{q}_{\perp k}\right) \\
M_{m, j+1}\left(p_{m}, p_{j}\right)=2 \pi \delta\left(p_{m}^{0}-p_{j}^{0}\right)\left(a_{m} \cdots a_{j+1}\right)(-i g)^{m-j}\left(2 E_{0}\right) e^{-i\left(p_{z m}-P_{\omega}\right) z_{m}} e^{-i\left(P_{\omega}-p_{z j}\right) z_{j+1}} \\
\quad \times \int \prod_{k=j+1}^{m}\left(\frac{d^{2} q_{\perp k}}{(2 \pi)^{2}} e^{-i \mathbf{q}_{\perp k} \cdot \mathbf{x}_{\perp k}} V_{k}^{a_{k}}\left(\mathbf{q}_{\perp k}\right)\right)(2 \pi)^{2} \delta\left(\mathbf{p}_{\perp m}-\mathbf{p}_{\perp j}-\sum_{k=j+1}^{m} \mathbf{q}_{\perp k}\right)
\end{gathered}
$$

We have utilized above that $p_{z 0}=P_{0} \approx E_{0}$ and that the energy of internal lines $k<j$ is $E_{0}$, while for $k \geq j$ it is $E_{0}-\omega$ with $p_{z k}=P_{\omega}=P_{0}-\omega / v_{0}$ on those lines. Note that the amplitude $M_{m, j+1}$ differs from the case of no radiation eq.(26) by only a $z$ dependent phase obtained by replacing $P_{0}$ with $P_{\omega}$ in eq.(27). In the eikonal limit eq. (53) can be expressed as

$$
\begin{aligned}
G_{j 1}^{c}= & 2 \pi \delta\left(p_{j}^{0}-p_{j-1}^{0}+\omega\right)\left[c, a_{j}\right](-i g)\left(2 E_{0}\right) e^{-i\left(p_{z j}-p_{z(j-1)}+k_{z}\right) z_{j}} \\
& \times \int \frac{d^{2} q_{\perp j}}{(2 \pi)^{2}} e^{-i \mathbf{q}_{\perp j} \cdot \mathbf{x}_{\perp j}} V_{j}^{a_{j}}\left(\mathbf{q}_{\perp j}\right)(2 \pi)^{2} \delta\left(\mathbf{q}_{\perp j}-\left(\mathbf{p}_{\perp j}-\mathbf{p}_{\perp(j-1)}+\mathbf{k}_{\perp}\right)\right) \\
& \times\left[\frac{-i \Gamma\left(k, p_{j}, p_{j-1}\right)}{\omega^{2}-\left(p_{z j}-p_{z(j-1)}\right)^{2}-\left(\mathbf{q}_{\perp j}-\mathbf{k}_{\perp}\right)^{2}}\right]
\end{aligned}
$$

where we used $-i T_{b} f_{b a_{j} c}=\left[c, a_{j}\right]$ and note that the vertex function is

$$
\begin{aligned}
\Gamma\left(k, p_{j}, p_{j-1}\right)= & 4 E_{0}\left(p_{j}-p_{j-1}\right) \epsilon(k)+4 \omega p_{j} \epsilon(k) \\
& -\left(p_{j}+p_{j-1}\right)\left(p_{j}-p_{j-1}+2 k\right) \epsilon^{0}(k) .
\end{aligned}
$$

Inserting these expressions into eq.(52), the integrals over the $p_{j-1}^{0}, p_{j}^{0}$ variables give rise again to an overall $\tilde{\delta}(m 1)$ factor eq. (36) and set $p_{j-1}^{0}=E_{0}$ and $p_{j}^{0}=E_{\omega}=$ $E_{0}-\omega$. Then as in eq.(111) we integrate the contour over $p_{z j}$ and $p_{z(j-1)}$ keeping only the residues at the poles of the propagators since $L_{j} \gg d$. Because of the three 
propagators involved, $\Delta\left(p_{j}\right) \Delta\left(p_{j}-p_{j-1}\right) \Delta\left(p_{j-1}\right)$, those integrals give rise to three contributions corresponding to forward scattering with two of the three internal lines set on shell. A fourth contribution corresponding to backscattering of the gluon near $z_{j}$ is suppressed in the kinematic range $E_{0} \gg \omega \gg \max \left(k_{\perp}, q_{\perp j}\right)$ of interest here. The kinematic variables of the three forward amplitudes after the contour integration are then as follows: In case $1, p_{j}^{2}=p_{j-1}^{2}=0,\left(p_{j}-p_{j-1}\right)^{2} \approx-\left(\mathbf{q}_{\perp j}-\mathbf{k}_{\perp}\right)^{2}$, with $\mathbf{p}_{\perp j}-\mathbf{p}_{\perp(j-1)}=\mathbf{q}_{\perp j}-\mathbf{k}_{\perp}$, and

$$
\begin{aligned}
p_{j-1} & =\left(E_{0}, E_{0}-p_{\perp(j-1)}^{2} / 2 E_{0}, \mathbf{p}_{\perp(j-1)}\right) \\
p_{j} & =\left(E_{0}-\omega, E_{0}-\omega-p_{\perp j}^{2} / 2 E_{0}, \mathbf{p}_{\perp j}\right) .
\end{aligned}
$$

In case $2,\left(p_{j}-p_{j-1}\right)^{2}=p_{j-1}^{2}=0, p_{j}^{2} \approx-\left(\mathbf{q}_{\perp j}-\mathbf{k}_{\perp}\right)^{2} / x$, and

$$
\begin{aligned}
p_{j-1} & =\left(E_{0}, E_{0}-p_{\perp(j-1)}^{2} / 2 E_{0}, \mathbf{p}_{\perp(j-1)}\right) \\
p_{j} & =\left(E_{0}-\omega, E_{0}-\omega+\left(\mathbf{q}_{\perp j}-\mathbf{k}_{\perp}\right)^{2} / 2 \omega, \mathbf{p}_{\perp j}\right) .
\end{aligned}
$$

Finally, in case $3\left(p_{j}-p_{j-1}\right)^{2}=p_{j}^{2}=0, p_{j-1}^{2} \approx\left(\mathbf{q}_{\perp j}-\mathbf{k}_{\perp}\right)^{2} / x$, and

$$
\begin{aligned}
p_{j-1} & =\left(E_{0}, E_{0}-\left(\mathbf{q}_{\perp j}-\mathbf{k}_{\perp}\right)^{2} / 2 \omega, \mathbf{p}_{\perp(j-1)}\right) \\
p_{j} & =\left(E_{0}-\omega, E_{0}-\omega-p_{\perp j}^{2} / 2 E_{0}, \mathbf{p}_{\perp j}\right) .
\end{aligned}
$$

We assume that $\omega^{2} \gg\left(\mathbf{q}_{\perp j}-\mathbf{k}_{\perp}\right)^{2}$. It is remarkable that the residue of the product of propagators is approximately same up to a sign in all three case with

$$
(-1) \operatorname{Res}\left(\Delta\left(p_{j}\right) \Delta\left(p_{j}-p_{j-1}\right) \Delta\left(p_{j-1}\right)\right) \approx \pm \frac{1}{\left(\mathbf{q}_{\perp j}-\mathbf{k}_{\perp}\right)^{2}\left(2 E_{0}\right)^{2}}
$$

with + for cases 1 and 2 and - for case 3 . Also the vertex factor turns out to be approximately the same in all three cases

$$
\Gamma \approx-4 E_{0} \vec{\epsilon}_{\perp} \cdot\left(\mathbf{q}_{\perp j}-\mathbf{k}_{\perp}\right)
$$

For a jet parton with mass, $\mu$, the singularity in eq.(132) at $\mathbf{q}_{\perp j}=\mathbf{k}_{\perp}$ is automatically regulated as in eq.(42) by

$$
\left|\mathbf{q}_{\perp j}-\mathbf{k}_{\perp}\right|^{-2} \rightarrow\left(\left|\mathbf{q}_{\perp j}-\mathbf{k}_{\perp}\right|^{2}+x^{2} \mu^{2}\right)^{-1}
$$


In higher order, the inclusion of the gluon proper self energy tensor in the medium would replace this infrared regulator by the effective dynamic mass of the gluons.

Only the $z$ dependent phase,

$$
\phi_{z}=-\left(P_{\omega}-p_{z j}\right) z_{j+1}-\left(p_{z j}-p_{z(j-1)}+k_{z}\right) z_{j}-\left(p_{z(j-1)}-P_{0}\right) z_{j-1}
$$

is found to be case dependent. Evaluating with eq.(129,130,131), we find that

$$
\phi=\left(\omega-k_{z}\right) z_{j}+ \begin{cases}0 & \text { case } 1 \\ L_{j} / \tau_{j} & \text { case } 2 \\ -L_{j-1} / \tau_{j} & \text { case } 3\end{cases}
$$

where $\tau_{j} \equiv 2 \omega /\left(\mathbf{q}_{\perp j}-\mathbf{k}_{\perp}\right)^{2}$ is the lifetime of the virtual jet line in cases 2 and 3 , and $L_{j}=z_{j+1}-z_{j}$ is the longitudinal distance between adjacent scattering centers. Together with the relative signs in eq.(132), the phase factors in the three cases sum to

$$
e^{i\left(\omega-k_{z}\right) z_{j}}\left(1+e^{i L_{j} / \tau_{j}}-e^{-i L_{j-1} / \tau_{j}}\right) \equiv e^{i z_{j} / \tau(k)} f_{j}(k)
$$

We therefore find a new interference form factor, $f_{j}$, that involves the separation distances between adjacent centers and the lifetime of the virtual jet state, $\tau_{j}$. For well separated centers, in the sense that the mean free path $\lambda=\left\langle\left(z_{i+1}-z_{i}\right)\right\rangle \gg \tau_{j}$, the extra phases in $f_{j}$ average to zero and $f_{j} \approx 1$. Also in the extreme opposite limit, $\lambda \ll \tau_{j}$, extra terms tend to cancel again leading to $f_{j} \approx 1$. In particular, for $\mathbf{k}_{\perp}=\mathbf{q}_{\perp j}, f_{j}=1$. As a rough form illustrating these limits,

$$
f_{j}(k) \sim 1+2 i \sin \left(\lambda\left(\mathbf{q}_{\perp j}-\mathbf{k}_{\perp}\right)^{2} / 2 \omega\right)
$$

In the general case, $f_{j} \neq 1$ reflects the effects of final state cascading of the emitted gluon. Another important limit is $q_{j}=0$ or $k_{\perp} \gg q_{\perp j}$. In this limit, $\tau_{j} \approx \tau(k)=$ $1 /\left(\omega-k_{z}\right)$, and $f_{j}$ reduces to

$$
f_{j} \approx 1+e^{i L_{j} / \tau(k)}-e^{i L_{j-1} / \tau(k)}
$$


For radiation with formation time much less or much longer than the mean free path, $f_{j} \approx 1$. Therefore, except in the restricted kinematic domain where $\tau_{j}$ or $\tau(k)$ is on the order of the mean free path, this extra interference effect can be neglected, and $f_{j}$ can be set to unity. Combining these results, we obtain eq.(56). 